\title{
A Joint Time-Invariant Filtering Approach to the Linear Gaussian Relay Problem
}

\author{
Cheulsoon Kim, Student Member, IEEE, Youngchul Sung, Senior Member, IEEE, and \\ Yong H. Lee, Senior Member, IEEE
}

\begin{abstract}
In this paper, the linear Gaussian relay problem is considered. Under the linear time-invariant (LTI) model the rate maximization problem in the linear Gaussian relay channel is formulated in the frequency domain based on the Toeplitz distribution theorem. Under the further assumption of realizable input spectra, the rate maximization problem is converted to the problem of joint source and relay filter design with two power constraints, one at the source and the other at the relay, and a practical solution to this problem is proposed based on the (adaptive) projected (sub)gradient method. Numerical results show that the proposed method yields a considerable gain over the instantaneous amplify-and-forward (AF) scheme in inter-symbol interference (ISI) channels. Also, the optimality of the AF scheme within the class of one-tap relay filters is established in flat-fading channels.
\end{abstract}

Index Terms-Filter design, linear Gaussian relay, linear timeinvariant model, projected subgradient method, Toeplitz distribution theorem.

\section{INTRODUCTION}

$\mathbf{R}$ ELAY networks have drawn extensive interest from the research community because they play an important role in enlarging the network coverage in wireless communications. Although the capacity of relay networks is not exactly known yet, many ingenious coding strategies including decode-and-forward (DF) and compress-and-forward (CF) beyond the simple AF scheme have been developed [1], [2]. Recently, Zahedi et al. proposed an advanced linear scheme for relay networks based on causal linear processing at the relay to compromise the complexity and performance between the complicated coding strategies and the simple AF scheme [3], [4]. (In this paper, the AF scheme means the instantaneous AF scheme which can easily be implemented by simple analog processing.) While information theorists approached the problem

Manuscript received August 08, 2011; revised December 15, 2011; accepted April 18, 2012. Date of publication May 04, 2012; date of current version July 10,2012 . The associate editor coordinating the review of this manuscript and approving it for publication was Prof. Eduard A. Jorswieck. This research was supported in part by the Basic Science Research Program through the National Research Foundation of Korea (NRF) funded by the Ministry of Education, Science and Technology (2010-0021269). This work was also supported in part by the IT R\&D program of MKE/KEIT [2008-F-004-02, "5G mobile communication systems based on beam division multiple access and relays with group cooperation"].

The authors are with the Department of Electrical Engineering, KAIST, 305-701 Daejeon, South Korea (e-mail: cs.kim@kaist.ac.kr; ysung@ee. kaist.ac.kr; yohlee@kaist.ac.kr).

This paper has supplementary downloadable multimedia material available at http://ieeexplore.ieee.org provided by the authors. This includes Matlab source files for the plots in the paper. This material is $86 \mathrm{~KB}$ in size.

Color versions of one or more of the figures in this paper are available online at http://ieeexplore.ieee.org.

Digital Object Identifier 10.1109/TSP.2012.2197750 from the perspective of capacity and capacity-achieving schemes [5]-[8], researchers in the signal-processing community also tackled this problem based on measures like the received signal-to-noise ratio (SNR) or minimum mean-square error (MMSE) [9]-[12]. Although these works provide meaningful approaches to the relay problem, they do not address the fundamental problem of data rate maximization directly. To maximize the data rate in the relay channel the processing at the source such as the input covariance function design should be incorporated together with the processing at the relay. However, the joint design of source and relay processing for rate maximization is a hard problem, as shown in [3] and [4]. While there exist some results on the rate-maximizing beamformer design in the case of multiple-input and multiple-output (MIMO) relay networks [13], [14], not much results are available for the general linear Gaussian relay channel. In [3] and [4], the authors considered general time-varying linear processing at the relay in the Gaussian relay channel. Although they obtained the capacity for frequency-division linear relaying, the general linear relay case was not explored fully [3], [4]. In the general linear relay case, the problem is a sequence of non-convex optimization problems, and it is seemingly intractable. To circumvent such difficulty, in this paper, we consider tractable and practical LTI filtering at the source and the relay, and provide a practical solution to design the source and relay filters jointly to maximize the transmission rate for general ISI Gaussian relay networks.

Under the LTI framework, the linear Gaussian relay problem can be formulated in the frequency domain using the Toeplitz distribution theorem [15], [16]. When the relay filter is given and there is no power constraint on the relay, the problem reduces to the classical ISI channel problem for which the optimal strategy is known as water-filling in the frequency domain [17, pp. 407-430]. However, the freedom to design the relay filter and the power constraint at the relay make the problem far more difficult than the classical ISI channel problem, especially when stability and causality constraints are imposed on the source and relay filters. Our approach to this problem is that we first convert the problem to a constrained optimization problem in a finite dimensional space by restricting the source and relay filters to the class of finite impulse response (FIR) filters as in most practical filtering applications, and then apply the (adaptive) projected (sub)gradient method, initially proposed by Goldstein [18], Polyak [19] and fully developed by Yamada et al. [20], [21], to this problem. The advantage of this approach is that the adaptive filtering principle can be applied to the filter design for rate maximization in the linear Gaussian relay channel and a very efficient adaptive implementation is possible in the case of realistic time-varying channels beyond static channels. Nu- 


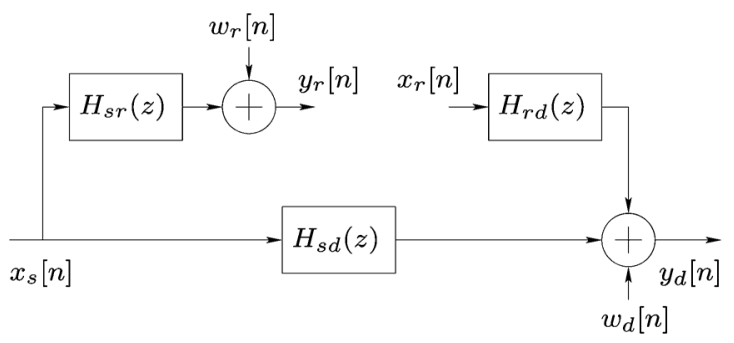

(a)

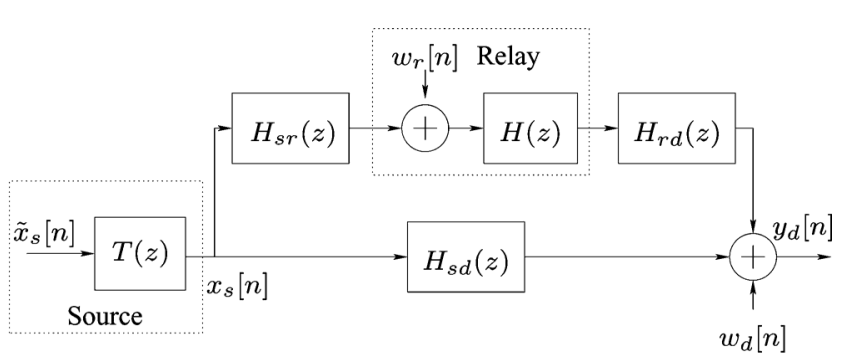

(b)

Fig. 1. (a) Channel model and (b) system model with linear time-invariant source and relay filters.

merical results show that our method performs well and yields a considerable gain over the AF scheme in ISI relay channels.

Notations and Organizations We will make use of standard notational conventions. Vectors and matrices are written in boldface with matrices in capitals. All vectors are column vectors. For a scalar $a, a^{*}$ denotes its complex conjugate. For a matrix $\mathbf{A}$, $\mathbf{A}^{T}, \mathbf{A}^{H}$ and $\operatorname{tr}(\mathbf{A})$ indicate the transpose, Hermitian transpose and trace of $\mathbf{A}$, respectively, and $\mathbf{A}(m, n)$ denotes the $m$ th row and $n$th column element of $\mathbf{A} \cdot \operatorname{diag}\left(d_{1}, \ldots, d_{n}\right)$ denotes a diagonal matrix with elements $d_{1}, \ldots, d_{n} . \mathbf{I}_{n}$ stands for the identity matrix of size $n$ (the subscript is omitted when unnecessary), and $\mathbf{0}$ denotes a vector of all zero elements. For a vector $\mathbf{a},\|\mathbf{a}\|$ denotes its 2-norm. The notation $\mathbf{x} \sim \mathcal{N}(\boldsymbol{\mu}, \boldsymbol{\Sigma})$ means that $\mathbf{x}$ is Gaussian-distributed with mean vector $\boldsymbol{\mu}$ and covariance matrix $\boldsymbol{\Sigma}$. $\mathbb{E}\{\cdot\}$ denotes the expectation. For two signal processes $x[n]$ and $y[n], x[n] * y[n]$ denotes the convolution of the two processes. $\mathbb{R}, \mathbb{\|}, \rrbracket_{+}$and $\mathbb{N}$ denote the sets of real numbers, integers, nonnegative integers and natural numbers, respectively. For two sets $A$ and $B, A \backslash B$ denotes the set minus operation. $j:=\sqrt{-1}$.

This paper is organized as follows. The system model and background are described in Section II. In Section III, the rate formula in the frequency domain is derived under the LTI model, and the performance of LTI relaying in flat-fading channels is investigated in Section IV. In Section V, a joint source and relay filter design method is proposed based on the (adaptive) projected (sub)gradient method, and its performance in ISI channels is examined in Section VI, followed by conclusions in Section VII.

\section{SyStem Model AND BACKGROUND}

We consider the general discrete-time additive white Gaussian noise (AWGN) relay network composed of source, relay and destination nodes, as shown in Fig. 1(a), where the source and relay nodes have maximum available average power $P_{s}$ and $P_{r}$, respectively. We assume that all propagation channels (i.e., the source-to-relay (S-R), relay-to-destination (R-D) and source-to-destination (S-D) channels) are linear, time-invariant and causal, and their impulse responses are absolutely summable, i.e., $\sum_{l=0}^{\infty}\left|h_{\mathrm{sr}}[l]\right|<\infty, \sum_{l=0}^{\infty}\left|h_{\mathrm{rd}}[l]\right|<\infty$ and $\sum_{l=0}^{\infty}\left|h_{\mathrm{sd}}[l]\right|<\infty$, where $h_{\mathrm{sr}}[l], h_{\mathrm{rd}}[l]$ and $h_{\mathrm{sd}}[l]$ are the S-R, R-D, and S-D channel impulse responses, respectively. Due to the absolute summability, the $z$-transforms of the propagation channel impulse responses are well defined and given by $H_{\mathrm{sr}}(z)=\sum_{l=0}^{\infty} h_{\mathrm{sr}}[l] z^{-l}, H_{\mathrm{rd}}(z)=\sum_{l=0}^{\infty} h_{\mathrm{rd}}[l] z^{-l}$ and $H_{\mathrm{sd}}(z)=\sum_{l=0}^{\infty} h_{\mathrm{sd}}[l] z^{-l}$. Then, the received signals at the relay and the destination at the $n$th symbol time are given by

$$
\begin{aligned}
& y_{r}[n]=h_{\mathrm{sr}}[n] * x_{s}[n]+w_{r}[n] \text { and } \\
& y_{d}[n]=h_{\mathrm{sd}}[n] * x_{s}[n]+h_{\mathrm{rd}}[n] * x_{r}[n]+w_{d}[n],
\end{aligned}
$$

respectively, where $x_{s}[n]$ is the transmitted signal process at the source; $x_{r}[n]$ and $y_{r}[n]$ are the transmitted and received signal processes at the relay, respectively; $y_{d}[n]$ is the received signal process at the destination; and the noise processes $w_{r}[n]$ at the relay and $w_{d}[n]$ at the destination are independent zero-mean white Gaussian processes with variance $\sigma^{2}$.

We consider the linear and causal processing at the relay. The general causal linear processing at the relay is given by

$$
x_{r}[n]=\sum_{l \leq n} d_{n l} y_{r}[l],
$$

for arbitrary linear combination coefficients $d_{n l}$, as considered in [3] and [4]. However, such linear processing requires timevarying filtering at the relay, and is not readily realizable. Thus, in this paper, we restrict ourselves to the case of LTI causal filtering at the relay, as shown in Fig. 1(b). In this case, the relay output is given by

$$
x_{r}[n]=\sum_{l=0}^{\infty} h_{l} y_{r}[n-l]
$$

where $\left[h_{0}, h_{1}, h_{2}, \ldots\right]$ is the time-invariant impulse response of the relay filter and its $z$-transform is given by $H(z)=\sum_{l=0}^{\infty} h_{l} z^{-l}$. (In the case of strict causality, we have $h_{0}=0$.) The signal model (3) at the relay can be written in matrix form as (4), and the filtering matrix $\mathbf{H}_{n}$ in (4) has a Toeplitz structure.

$$
\left[\begin{array}{c}
x_{r}[0] \\
x_{r}[1] \\
\vdots \\
x_{r}[n-1]
\end{array}\right]=\underbrace{\left[\begin{array}{cccc}
h_{0} & 0 & \cdots & 0 \\
h_{1} & h_{0} & 0 & \\
\vdots & \ddots & \ddots & \vdots \\
h_{n-1} & \cdots & h_{1} & h_{0}
\end{array}\right]}_{=: \mathbf{H}_{n}}\left[\begin{array}{c}
y_{r}[0] \\
y_{r}[1] \\
\vdots \\
y_{r}[n-1]
\end{array}\right] .
$$

We assume the stability (i.e., $\sum_{l=0}^{\infty}\left|h_{l}\right|<\infty$ ) for the relay filter. Since all processing from the source to the destination is linear and time-invariant, the received signal at the destination in the $z$-domain is given by

$$
\begin{array}{r}
Y_{d}(z)=\left(H_{\mathrm{sd}}(z)+H_{\mathrm{rd}}(z) H(z) H_{\mathrm{sr}}(z)\right) X_{s}(z) \\
+H_{\mathrm{rd}}(z) H(z) W_{r}(z)+W_{d}(z)
\end{array}
$$

where $X_{s}(z)$ is the $z$-transform of the source process, and $W_{r}(z)$ and $W_{d}(z)$ are the $z$-transforms of noise processes $w_{r}[n]$ and $w_{d}[n]$, respectively.

\section{A. Background}

In this subsection, we briefly summarize some relevant results including the eigenstructure of Toeplitz matrices and the spectral factorization for the development in later sections. For 
a zero-mean ${ }^{1}$ stationary random process $y[n]$, its covariance sequence $r_{y}[k]$ and $z$-spectrum $S_{y}(z)$ are given by

$$
\begin{aligned}
r_{y}[k] & =\mathbb{E}\left\{y[n] y^{*}[n-k]\right\}=r_{y}^{*}[-k] \text { and } \\
S_{y}(z) & :=\sum_{k=-\infty}^{\infty} r_{y}[k] z^{-k}
\end{aligned}
$$

respectively. The covariance matrix of a finite collection $\mathbf{y}_{n}:=$ $[y[0], y[1], \ldots, y[n-1]]^{T}$ is given by

$$
\Sigma_{n}^{y}:=\mathbb{E}\left\{\mathbf{y}_{n} \mathbf{y}_{n}^{H}\right\}=\left[\begin{array}{cccc}
r_{y}[0] & r_{y}[-1] & \cdots & r_{y}[-n+1] \\
r_{y}[1] & r_{y}[0] & & \vdots \\
\vdots & \vdots & \ddots & r_{y}[-1] \\
r_{y}[n-1] & r_{y}[n-2] & \cdots & r_{y}[0]
\end{array}\right]_{(7)}
$$

Based on the asymptotic eigenstructure of Toeplitz covariance matrices, we have the following theorem.

Theorem 1 (Toeplitz Distribution Theorem [15, p. 65]): Let $r_{y}[k]$ be an absolutely summable autocovariance sequence of a stationary process $y[n]$, let $S_{y}\left(e^{j \omega}\right)$ be its power spectral density (PSD), i.e., $S_{y}\left(e^{j \omega}\right)=\left.S_{y}(z)\right|_{z=e^{j \omega}}$, and let $\left\{\lambda_{i}^{(n)}\right\}$ be the eigenvalues of the Toeplitz covariance matrix $\boldsymbol{\Sigma}_{n}^{y}$ of the stationary process $y[n]$. Then,

$$
\lim _{n \rightarrow \infty} \frac{1}{n} \sum_{i=1}^{n} f\left(\lambda_{i}^{(n)}\right)=\frac{1}{2 \pi} \int_{-\pi}^{\pi} f\left(S_{y}\left(e^{j \omega}\right)\right) d \omega
$$

for any continuous function $f(\cdot)$.

Theorem 1 is based on the fact that the eigenvalues of a Toeplitz covariance matrix converge to the uniform samples of its spectrum for sufficiently large $n$ [16, p. 135], and states that the normalized sum of the eigenvalues of a Toeplitz covariance matrix converges to the integral of its spectrum and this is valid even for any continuous mapping of the eigenvalues. This theorem will be used to obtain an asymptotic rate formula in the frequency domain in later sections. In addition to the Toeplitz distribution theorem, we need some background in the spectral theory for stationary random processes, especially canonical spectral factorization.

Definition 1 (Canonical Spectral Factorization [22, p. 197]): Let $S_{y}(z)$ be a rational $z$-spectrum of a finite power process and assume that $S_{y}(z)$ is strictly positive. Then, the canonical spectral factorization of $S_{y}(z)$ is given by $S_{y}(z)=L(z) \gamma_{e} L^{\sharp}(z)$, where $L(z)=\sum_{i=0}^{\infty} l_{i} z^{-i}$ is a unique stable, causal, monic and minimum-phase (SCAMP) filter (i.e., the zeros and poles of $L(z)$ are strictly inside the unit circle and $L(\infty)=1$ (or equivalently $\left.l_{0}=1\right)$ ), and $\gamma_{e}>0$. Here, $L^{\sharp}(z):=L^{*}\left(z^{-*}\right)$ denotes the para-Hermitian conjugate.

\section{The RATE Formula IN FREQUENCY-DOMAIN FOR LTI RELAYS}

First, note that the overall channel model (5) with LTI relay filtering is still a linear additive stationary Gaussian noise channel. Thus, for a given relay filter, the overall channel with the LTI relay filter reduces back to the classical ISI channel with stationary Gaussian noise. ${ }^{2}$ In this case, stationary Gaussian

\footnotetext{
${ }^{1}$ In the case of a known non-zero mean, the mean of the process can be subtracted and the result can still be applied.

${ }^{2}$ However, the major difference between the two problems is that in the relay problem we even have to design the overall channel by properly choosing the relay filter and the power constraints at the source and relay are intertwined.
}

signal processes with well-defined spectra are sufficient to achieve the capacity [17, pp. 407-430]. Hence, we assume that the source (or input) process $x_{s}[n]$ is a stationary Gaussian process. By concatenating symbols at the source up to time $n-1$, we have

$$
\mathbf{x}_{n}^{s}:=\left[x_{s}[0], x_{s}[1], \ldots, x_{s}[n-1]\right]^{T} \sim \mathcal{N}\left(\mathbf{0}, \boldsymbol{\Sigma}_{n}^{x_{s}}\right),
$$

and vectors $\mathbf{y}_{n}^{r}, \mathbf{x}_{n}^{r}$ and $\mathbf{y}_{n}^{d}$ are constructed similarly for the relay and destination nodes. Then, the power constraints for the source and relay are respectively given by

$$
\begin{aligned}
& \left(\frac{1}{n}\right) \operatorname{tr}\left(\mathbf{\Sigma}_{n}^{x_{s}}\right) \leq P_{s}, \text { and } \\
& \left(\frac{1}{n}\right) \mathbb{E}\left\{\operatorname{tr}\left(\mathbf{H}_{n} \mathbf{y}_{n}^{r}\left(\mathbf{H}_{n} \mathbf{y}_{n}^{r}\right)^{H}\right)\right\}= \\
& \left.\left(\frac{1}{n}\right) \operatorname{tr}\left(\mathbf{H}_{n}\left\{\mathbf{H}_{n}^{\mathrm{sr}} \boldsymbol{\Sigma}_{n}^{x_{s}}\left(\mathbf{H}_{n}^{\mathrm{sr}}\right)^{H}+\sigma^{2} \mathbf{I}\right)\right\} \mathbf{H}_{n}^{H}\right) \leq P_{r}
\end{aligned}
$$

where $\mathbf{H}_{n}^{\text {sr }}$ is the filtering matrix for the S-R channel constructed based on $\left\{h_{\mathrm{sr}}[l]\right\}$ similar to $\mathbf{H}_{n}$ in (4). Thus, the maximum rate with LTI relaying for block size $n$ is given by maximizing the mutual information between $\mathbf{x}_{n}^{s}$ and $\mathbf{y}_{n}^{d}$ over $\boldsymbol{\Sigma}_{n}^{x_{s}}$ and $\mathbf{H}_{n}$ under power constraints (10) and (11), and the capacity with LTI relaying is given by its limit

$$
C_{\mathrm{LTI}}=\lim _{n \rightarrow \infty} \sup _{\mathbf{\Sigma}_{n}^{\mathbf{x}_{s}}, \mathbf{H}_{n}} \frac{1}{n} I\left(\mathbf{x}_{n}^{s} ; \mathbf{y}_{n}^{d}\right),
$$

as $n \rightarrow \infty$, if the limit exists [4], where

$$
\begin{aligned}
& I\left(\mathbf{x}_{n}^{s} ; \mathbf{y}_{n}^{d}\right)= H\left(\mathbf{y}_{n}^{d}\right)-H\left(\mathbf{y}_{n}^{d} \mid \mathbf{x}_{n}^{s}\right), \\
&=\log \mid \sigma^{2} \mathbf{H}_{n}^{r d} \mathbf{H}_{n} \mathbf{H}_{n}^{H}\left(\mathbf{H}_{n}^{r d}\right)^{H}+\sigma^{2} \mathbf{I} \\
& \quad+\left(\mathbf{H}_{n}^{s d}+\mathbf{H}_{n}^{r d} \mathbf{H}_{n} \mathbf{H}_{n}^{\mathrm{sr}}\right) \\
& \times \mathbf{\Sigma}_{n}^{x_{s}}\left(\mathbf{H}_{n}^{s d}+\mathbf{H}_{n}^{r d} \mathbf{H}_{n} \mathbf{H}_{n}^{\mathrm{sr}}\right)^{H} \mid \\
&-\log \left|\sigma^{2} \mathbf{H}_{n}^{r d} \mathbf{H}_{n} \mathbf{H}_{n}^{H}\left(\mathbf{H}_{n}^{r d}\right)^{H}+\sigma^{2} \mathbf{I}\right| .
\end{aligned}
$$

Here, $\mathbf{H}_{n}^{s d}$ and $\mathbf{H}_{n}^{r d}$ are the filtering matrices for the S-D and R-D channels, respectively. Note that (12) is still valid for general linear time-varying relay filtering with $\mathbf{H}_{n}$ given by an arbitrary lower triangular matrix. As mentioned in [4], the computation of capacity and the design of capacity-achieving (or at least reasonable) $\boldsymbol{\Sigma}_{n}^{x_{s}}$ and $\mathbf{H}_{n}$ are difficult problems in the case of general linear causal relay filtering. In the time-varying case, if we increase $n$ by one, at least $2 n$ new variables $\left\{\boldsymbol{\Sigma}_{n}^{x_{s}}(n, 1), \boldsymbol{\Sigma}_{n}^{x_{s}}(n, 2), \ldots, \boldsymbol{\Sigma}_{n}^{x_{s}}(n, n), d_{n 1}, d_{n 2}, \ldots, d_{n n}\right\}$ appear (see (2)), and thus the complexity of the problem increases with the order of $n$ ! to make the problem difficult [3], [4]. In the LTI case with a stationary source process, however, we have only two new variables $r_{x_{s}}[n-1]$ and $h_{n-1}$ for the increase of the problem size from $n-1$ to $n$ because of the Toeplitz structure of the covariance matrix in (7) and the filtering matrix in (4). Following the best input covariance matrix and relay filter for the problem size $n$ is equivalent to designing the best infinitely long autocovariance sequence $\left\{r_{x_{s}}[k], k=0,1, \ldots\right\}$ and infinitely long relay filter $\left\{h_{l}, l=0,1, \ldots\right\}$ first and then increasing the problem size. Thus, in the LTI case, we have

$$
\sup _{\left.\operatorname{LrTI}_{x_{s}}[k]\right\},\left\{h_{l}\right\}} \lim _{n \rightarrow \infty} \frac{1}{n}\left[I\left(\mathbf{x}_{n}^{s} ; \mathbf{y}_{n}^{d}\right) \mid \mathbf{\Sigma}_{n}^{x_{s}}\left(\left\{r_{x_{s}}[k]\right\}\right), \mathbf{H}_{n}\left(\left\{h_{l}\right\}\right)\right]
$$


where the respective dependence of $\boldsymbol{\Sigma}_{x}^{n}$ and $\mathbf{H}_{n}$ on the input signal autocovariance sequence $\left\{r_{x_{s}}[k]\right\}$ and the relay impulse response $\left\{h_{l}\right\}$ is explicitly shown. Here, taking the limit of $n$ simplifies the problem significantly due to Theorem 1 . The matrices inside the determinants in (13) are positive-definite Hermitian and thus each determinant is given by the product of the eigenvalues of the matrix inside the determinant. By changing the order of the logarithm and the product, we have the form in the left-hand side (LHS) of (8) with $f(t)=\log (t)$. Since the eigenvalues are strictly positive due to the additive noise term and since $f(t)=\log (t)$ is a continuous function of $t$ for $t>0$, Theorem 1 can be applied to yield (15) at the bottom of the page, where the input spectrum $S_{x_{s}}\left(e^{j \omega}\right)=\sum_{k=-\infty}^{\infty} r_{x_{s}}[k] e^{-j \omega k}$ and the relay response $H(z)=\sum_{l=0}^{\infty} h_{l} z^{-l}$. (The corresponding spectrum is easily obtained by the spectral property of linear systems.) Here, we define the overall channel-to-noise power ratio (CNR) density as

$$
\begin{aligned}
\operatorname{CNR}\left(e^{j \omega}\right): & :=\frac{\left|H_{\mathrm{sd}}\left(e^{j \omega}\right)+H_{\mathrm{sr}}\left(e^{j \omega}\right) H\left(e^{j \omega}\right) H_{\mathrm{rd}}\left(e^{j \omega}\right)\right|^{2}}{\sigma^{2}\left(\left|H_{\mathrm{rd}}\left(e^{j \omega}\right) H\left(e^{j \omega}\right)\right|^{2}+1\right)} \\
& =\frac{N\left(e^{j \omega}\right)}{D\left(e^{j \omega}\right)}
\end{aligned}
$$

where $N\left(e^{j \omega}\right)$ and $D\left(e^{j \omega}\right)$ are the numerator and denominator of the CNR density, respectively. Note that the CNR density captures the overall channel response from source to destination. When the CNR density is multiplied by the input signal PSD, the product becomes the overall SNR density at the destination. (This quantity will be used in later sections.) In addition to the rate formula (15) in the frequency domain, the power constraints can also be expressed in the frequency domain as $n \rightarrow \infty$. As $n \rightarrow \infty$, again by Theorem 1 , the power constraints (10) and (11) are respectively given by

$$
\frac{1}{2 \pi} \int_{-\pi}^{\pi} S_{x_{s}}\left(e^{j \omega}\right) d \omega \leq P_{s}, \text { and }
$$

$$
\frac{1}{2 \pi} \int_{-\pi}^{\pi}\left|H\left(e^{j \omega}\right)\right|^{2}\left(\left|H_{\mathrm{sr}}\left(e^{j \omega}\right)\right|^{2} S_{x_{s}}\left(e^{j \omega}\right)+\sigma^{2}\right) d \omega \leq P_{r}
$$

since the trace of a matrix is the sum of its eigenvalues. Thus, the rate maximization problem in the LTI Gaussian relay channel is summarized by (15), (17), and (18). Note that for a given relay filter $H(z)$ the problem without the power constraint (18) reduces to the well-known ISI channel problem and the solution of $S_{x_{s}}\left(e^{j \omega}\right)$ is given by water-filling in the frequency domain [23]. However, the freedom to design $H(z)$ and the relay power constraint (18) make the problem far more difficult than the simple ISI channel problem. To construct a practical method to solve this problem, we further assume that the input spectrum
$S_{x_{s}}\left(e^{j \omega}\right)$ is realizable. That is, its canonical spectral factorization is given by

$$
\begin{aligned}
S_{x_{s}}(z) & =\alpha \tilde{T}(z) \tilde{T}^{\sharp}(z)=T(z) T^{\sharp}(z), \\
(T(z) & =\sqrt{\alpha} \tilde{T}(z))
\end{aligned}
$$

where the SCAMP filter $\tilde{T}(z)$ has a rational transfer function and, thus, $S_{x_{s}}(z)$ is a rational spectrum. In this case, the source process $x_{s}[n]$ can be modelled as the output of the stable and causal autoregressive moving-average (ARMA) filter $T(z)$ driven by a white Gaussian process $\tilde{x}_{s}[n]$ with unit variance, as seen in Fig. 1(b). Thus, the rate maximization problem under LTI relaying with realizable input spectra now reduces to a joint design problem of LTI source and relay filters. We will propose a very efficient and practical solution to this joint filter design problem in general ISI relay channels in Section V. Before we tackle this problem, we investigate the problem in the case that all S-D, S-R and R-D channels have flat frequency responses in Section IV.

\section{EXAMINATION OF LTI RELAYING IN FLAT-FADING CHANNELS}

In the case of flat fading, we have the system model (5) in which each of S-R, R-D, and S-D channels has only one tap, i.e., $H_{\mathrm{sd}}(z)=1, H_{\mathrm{sr}}(z)=a$ and $H_{\mathrm{rd}}(z)=b$, as considered in [3], [4]. Then, the received signal model in the $z$-domain is given by

$$
Y_{d}(z)=(1+a b H(z)) X_{s}(z)+b H(z) W_{r}(z)+W_{d}(z) .
$$

\section{A. The One-Tap Relay Filter Case}

First, consider the well-known AF relaying. In this case, we have $x_{r}[n]=d y_{r}[n]$, where $\mathbb{E}\left\{x_{s}^{2}\right\}=P_{s}$ and $0 \leq d \leq$ $\sqrt{\frac{P_{r}}{a^{2} P_{s}+\sigma^{2}}}=: d_{\max }$ to satisfy the power constraints, and the received signal model is given by

$$
y_{d}[n]=(1+a b d) x_{s}[n]+b d w_{r}[n]+w_{d}[n] .
$$

Due to the simple data model (21), the achievable rate in this case is known and given by

$$
R_{\mathrm{AF}}=\max _{0 \leq d \leq d_{\max }} \frac{1}{2} \log \left(1+\frac{(1+a b d)^{2}}{b^{2} d^{2}+1} \cdot \frac{P_{s}}{\sigma^{2}}\right),
$$

and the optimal value of $d$ is explicitly given by

$$
d^{*}=\min \left\{\frac{a}{b}, \sqrt{\frac{P_{r}}{a^{2} P_{s}+\sigma^{2}}}\right\} .
$$

Now consider the one-tap LTI relay filter with an arbitrary delay, $H(z)=d z^{-\Delta}$, for some integer $\Delta \geq 0$, where the relay

$$
C_{\mathrm{LTI}}=\sup _{S_{x_{s}}\left(e^{j \omega}\right), H(z)} \frac{1}{2 \pi} \int_{-\pi}^{\pi} \frac{1}{2} \log _{2}\left(1+\frac{\left|H_{\mathrm{sd}}\left(e^{j \omega}\right)+H_{\mathrm{sr}}\left(e^{j \omega}\right) H\left(e^{j \omega}\right) H_{\mathrm{rd}}\left(e^{j \omega}\right)\right|^{2}}{\sigma^{2}\left(\left|H_{\mathrm{rd}}\left(e^{j \omega}\right) H\left(e^{j \omega}\right)\right|^{2}+1\right)} S_{x_{s}}\left(e^{j \omega}\right)\right) d \omega
$$


gain $d$ can be optimized under the power constraints. Note in the system model (20) that the relay filter affects both the channel gain and the noise spectrum. However, in the one-tap relay filter case, the problem is simplified because the overall noise spectrum is white. In this case, the overall channel gain is given by $1+a b H(z)=1+a b d z^{-\Delta}$ and the overall noise spectrum is given by

$b^{2} H(z) H^{\sharp}(z) \sigma^{2}+\sigma^{2}=b^{2} \sigma^{2}\left(d z^{-\Delta}\right)\left(d z^{-\Delta}\right)^{\sharp}+\sigma^{2}=\left(b^{2} d^{2}+1\right) \sigma^{2}$

since $\left(z^{-\Delta}\right)^{\sharp}=z^{\Delta}$. Note that the overall noise process in this case is white and equivalent to that in the AF data model (21); both have the same variance $\left(b^{2} d^{2}+1\right) \sigma^{2}$. Thus, the spectrum of $Y_{d}(z)$ is given by

$$
S_{y_{d}}\left(e^{j \omega}\right)=\left|1+a b H\left(e^{j \omega}\right)\right|^{2} S_{x_{s}}\left(e^{j \omega}\right)+\left(b^{2} d^{2}+1\right) \sigma^{2},
$$

and the channel frequency response is explicitly given by a raised-cosine function: $\left|1+a b H\left(e^{j \omega}\right)\right|^{2}=\left(1+a b d e^{-j \omega \Delta}\right)(1+$ $\left.a b d e^{j \omega \Delta}\right)=1+2 a b d \cos (\omega \Delta)+a^{2} b^{2} d^{2} \geq 0$. Since $\left|H\left(e^{j \omega}\right)\right|^{2}=d^{2}$ for $H(z)=d z^{-\Delta}$, from (17) and (18) the power constraints are given by

$$
\begin{aligned}
& \frac{1}{2 \pi} \int_{-\pi}^{\pi} S_{x_{s}}\left(e^{j \omega}\right) d \omega \leq P_{s}, \text { and } \\
& \frac{d^{2}}{2 \pi} \int_{-\pi}^{\pi}\left(a^{2} S_{x_{s}}\left(e^{j \omega}\right)+\sigma^{2}\right) d \omega \\
& \quad=d^{2}\left(a^{2} \frac{1}{2 \pi} \int_{-\pi}^{\pi} S_{x_{s}}\left(e^{j \omega}\right) d \omega+\sigma^{2}\right) \leq P_{r}
\end{aligned}
$$

which are the same as those of the AF scheme.

The problem with the given relay filter $H(z)=d z^{-\Delta}$ reduces to the simple ISI channel problem, and the optimal input spectrum $S_{x_{s}}^{*}\left(e^{j \omega}\right)$ is obtained by water-filling under the two simple power constraints (25) and (26). In the following theorem, we establish the optimality of the AF scheme within the class of all one-tap relay filters.

Theorem 2: Among all one-tap linear relay filters, i.e., $H(z)=d z^{-\Delta}$ with $\Delta \in \mathbb{\prod}$, the AF scheme with $\Delta=0$ maximizes the achievable rate.

Proof: For a given $\Delta \neq 0$, let

$$
\begin{aligned}
& \left(S_{x_{s}}^{*}\left(e^{j \omega}\right), d^{*}\right) \\
& =\underset{S_{x_{s}}\left(e^{j \omega}\right), d}{\arg \max } \frac{1}{2 \pi} \int_{-\pi}^{\pi} \frac{1}{2} \log \left(1+\frac{\left|1+a b d e^{-j \omega \Delta}\right|^{2}}{\left(b^{2} d^{2}+1\right) \sigma^{2}} S_{x_{s}}\left(e^{j \omega}\right)\right) d \omega .
\end{aligned}
$$

Then,

$$
\begin{aligned}
& \frac{1}{2 \pi} \int_{-\pi}^{\pi} \frac{1}{2} \log \left(1+\frac{\left|1+a b d^{*} e^{-j \omega \Delta}\right|^{2}}{\left(b^{2} d^{* 2}+1\right) \sigma^{2}} S_{x_{s}}^{*}\left(e^{j \omega}\right)\right) d \omega \\
& \leq \frac{1}{2 \pi} \int_{-\pi}^{\pi} \frac{1}{2} \log \left(1+\frac{\left(1+a b d^{*}\right)^{2}}{\left(b^{2} d^{* 2}+1\right) \sigma^{2}} S_{x_{s}}^{*}\left(e^{j \omega}\right)\right) d \omega \\
& \leq \sup _{S_{x_{s}}\left(e^{j \omega}\right), d} \frac{1}{2 \pi} \int_{-\pi}^{\pi} \frac{1}{2} \log \left(1+\frac{(1+a b d)^{2}}{\left(b^{2} d^{2}+1\right) \sigma^{2}} S_{x_{s}}\left(e^{j \omega}\right)\right) d \omega
\end{aligned}
$$

$$
\begin{aligned}
& \leq \sup _{S_{x_{s}}\left(e^{j \omega}\right), d} \frac{1}{2} \log \left(1+\frac{(1+a b d)^{2}}{\left(b^{2} d^{2}+1\right) \sigma^{2}} \frac{1}{2 \pi} \int_{-\pi}^{\pi} S_{x_{s}}\left(e^{j \omega}\right) d \omega\right) \\
& =R_{\mathrm{AF}} .
\end{aligned}
$$

Here, (28) is obtained because $\left|1+a b d^{*} e^{-j \omega \Delta}\right|^{2} \leq\left(1+a b d^{*}\right)^{2}$. Equation (29) is obtained because the feasible set $\left(S_{x_{s}}\left(e^{j \omega}\right), d\right)$ satisfying the power constraint for $\Delta \neq 0$ is the same as that for $\Delta=0$ when $H(z)=d z^{-\Delta}$. (See (25) and (26).) (30) is obtained by Jensen's inequality. Finally, (31) is obtained by the definition of $R_{\mathrm{AF}}$ in (22).

Theorem 2 states that the AF scheme with $\Delta=0$ performs best within the class of one-tap relay filters with arbitrary delays. This is because the AF scheme achieves coherent signal combining between the two signal paths S-D and S-R-D. Instead of using the optimal water-filling source filter, we can also consider a simple channel-equalizing source filter. However, the performance in this case is bad, as shown in the following theorem.

Theorem 3: The achievable rate by an equalizing source filter for the one-tap relay filter $H(z)=d z^{-\Delta}$ is given by

$$
\begin{aligned}
& R_{1-t a p, E Q} \\
& =\sup _{0 \leq d<\min \left\{d_{\max }, \frac{1}{a b}\right\}} \frac{1}{2} \log \left(1+\frac{1-(a b d)^{2}}{b^{2} d^{2}+1} \cdot \frac{P_{s}}{\sigma^{2}}\right)
\end{aligned}
$$

regardless of the value of $\Delta>0$. Further, the supremum is given by $R_{1-t a p, E Q}=\frac{1}{2} \log \left(1+\frac{P_{s}}{\sigma^{2}}\right)$ achieved when $d=0$.

Proof: We have $X_{s}(z)=\sqrt{\alpha} \hat{T}(z) \tilde{X}_{s}(z)$, where $\tilde{X}_{s}(z)$ is the $z$-transform of the white Gaussian process $\tilde{x}_{s}[n]$ with unit variance, and the equalizing source filter is given by

$$
\begin{aligned}
\tilde{T}(z) & =\frac{1}{1+a b H(z)}=\frac{1}{1+a b d z^{-\Delta}} \\
& =1-a b d z^{-\Delta}+(a b d)^{2} z^{-2 \Delta}-(a b d)^{3} z^{-3 \Delta}+\cdots .
\end{aligned}
$$

When $0 \leq d<\frac{1}{a b}$, the overall channel response $1+a b H(z)$ is SCAMP and, thus, the channel-equalizing source filter $\tilde{T}(z)$ is also SCAMP. By the power constraint at the source, we have

$$
\begin{aligned}
\frac{1}{2 \pi} \int_{-\pi}^{\pi} S_{x_{s}}\left(e^{j \omega}\right) d \omega & =\frac{\alpha}{2 \pi} \int_{-\pi}^{\pi} \frac{1}{1+2 a b d \cos (\omega \Delta)+(a b d)^{2}} d \omega \\
& =P_{s}
\end{aligned}
$$

because $S_{x_{s}}(z)=\alpha \tilde{T}(z) \tilde{T}^{\sharp}(z)$. Since

$$
\int_{-\pi}^{\pi} \frac{1}{1+2 a b d \cos (\omega \Delta)+(a b d)^{2}} d \omega=\frac{2 \pi}{1-(a b d)^{2}}
$$

for every integer $\Delta>0, \alpha=\left(1-(a b d)^{2}\right) P_{s}$ regardless of the value of $\Delta>0$. With the channel-equalizing source filter $T(z)=\sqrt{\alpha} \tilde{T}(z)$, the data model is given by $y_{d}[n]=$ $\sqrt{\alpha} \tilde{x}_{s}[n]+w_{\text {eff }}[n]$, where $\tilde{x}_{s}[n] \sim \mathcal{N}(0,1)$ and $w_{\text {eff }}[n] \sim$ $\mathcal{N}\left(0,\left(b^{2} d^{2}+1\right) \sigma^{2}\right)$, and the corresponding achievable rate is given by (32). Now consider $\operatorname{CNR}(d)=\frac{1-(a b d)^{2}}{b^{2} d^{2}+1} \frac{1}{\sigma^{2}}$ in (32). Its derivative with respect to (w.r.t.) $d$ is given by $\operatorname{CNR}^{\prime}(d)=$ $\frac{\left[-2 a^{2} b^{2} d\left(b^{2} d^{2}+1\right)-\left(1-a^{2} b^{2} d^{2}\right) 2 b^{2} d\right]}{\left[\left(b^{2} d^{2}+1\right)^{2} \sigma^{2}\right]} \leq 0$ for all $d \geq 0$. Thus, the rate is maximized when $d=0$. 


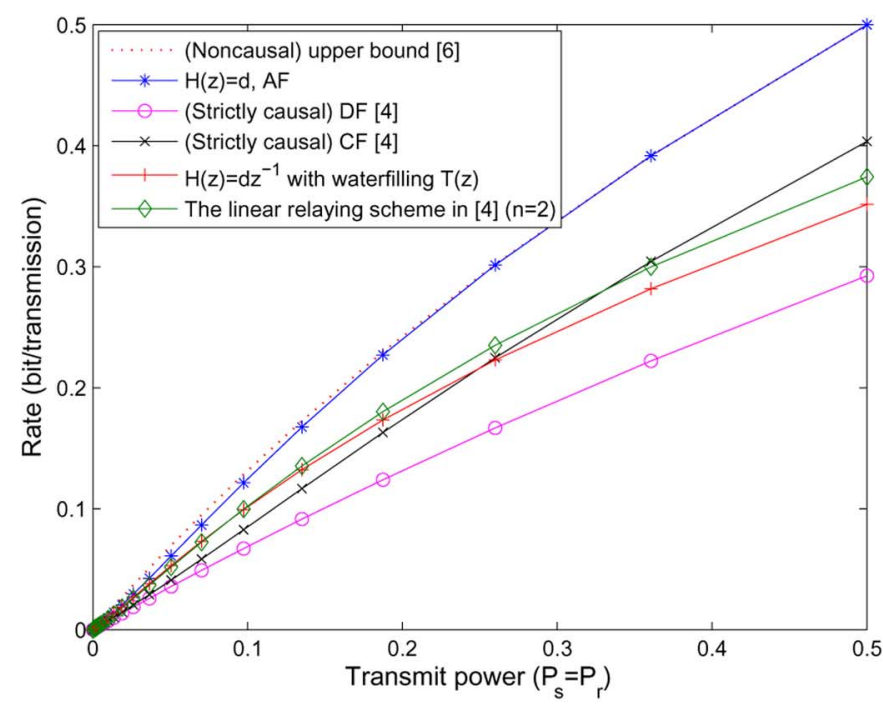

(a)

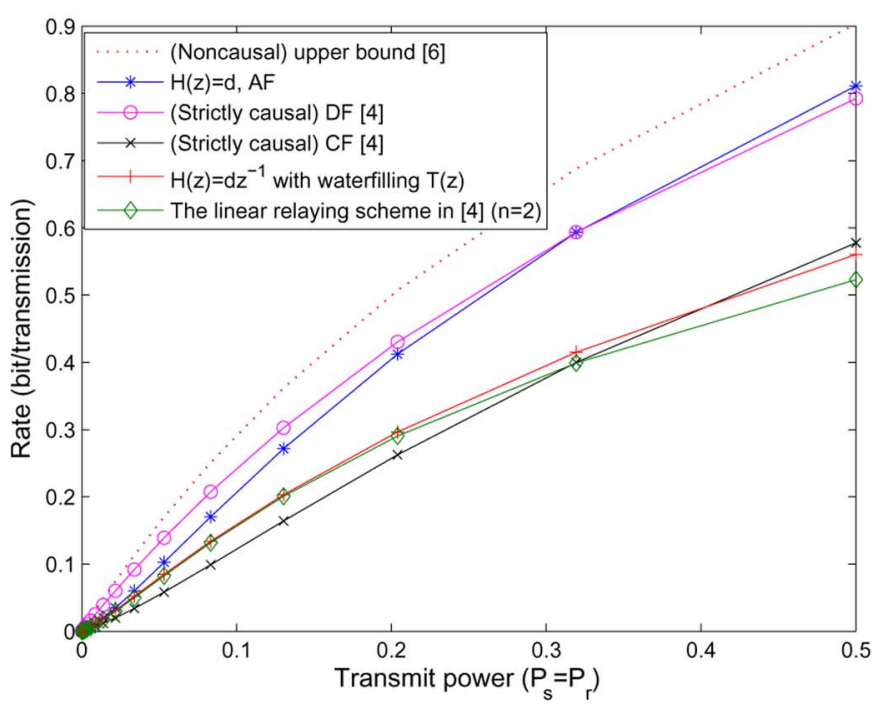

(c)

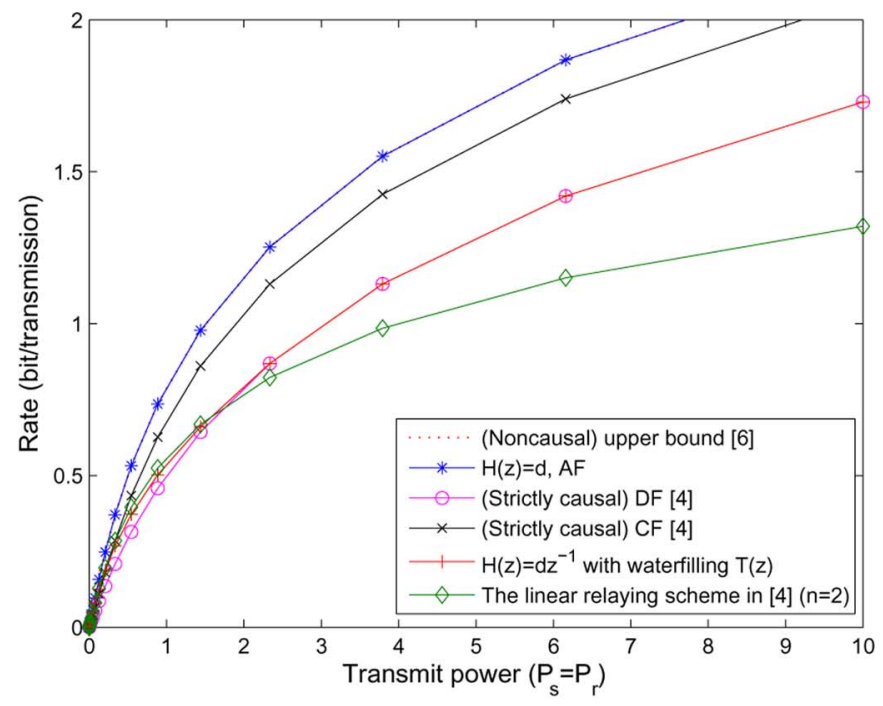

(b)

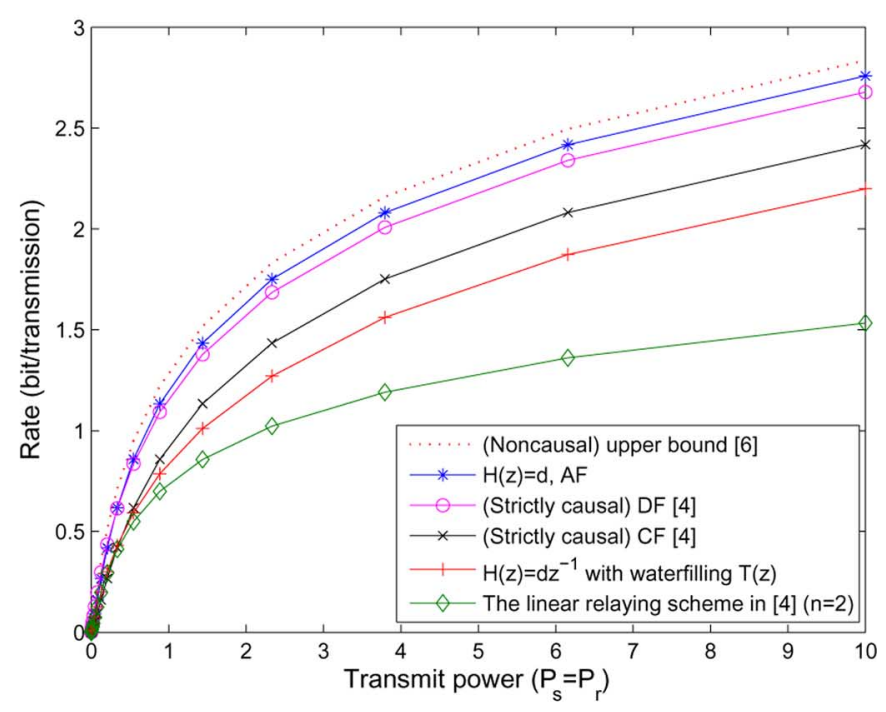

(d)

Fig. 2. Achievable rates of several schemes in flat-fading channels $\left(P_{\mathrm{s}}=P_{r}, \sigma^{2}=1\right)-(\mathrm{a})$ and (b): $a=1, b=2$, and (c) and (d): $a=2, b=2$.

Theorem 3 states that it is optimal to turn off the (one-tap) relay filter when the channel-equalizing filter is to be used at the source. Thus, using the channel-equalizing source filter is not a proper choice for relay networks. Fig. 2 shows the achievable rates of several relaying schemes when $P_{s}=P_{r}$. For Fig. 2(a) and (b), which show the same curves with two different $x$-axis ranges, we set $a=1$ and $b=2$, as in [4]. It is seen that simple linear strictly causal schemes (one based on the filtering matrix $\left[\begin{array}{ll}0 & 0 \\ d & 0\end{array}\right]$ in [4] and the other based on one-tap filtering $H(z)=d z^{-1}$ ) can outperform the CF scheme in the low SNR region, as already known from [4]. In this case of $a=1$ and $b=2$, the AF scheme achieves the upper bound for $P_{s}=P_{r} \geq \frac{1}{3}$ [6, Proposition 9]. It is interesting to observe that the simple linear scheme in [4] with $n=2$ performs better than $H(z)=d z^{-1}$ filtering in some low SNR values, although the latter outperforms the former eventually at high SNR. Fig. 2(c) and (d), again showing the same curves in two different $x$-axis ranges, show the achievable rates when $a=b=2$. In this case, it is seen that there is a gap between the upper bound and the AF scheme. In all the cases, it is seen that the two strictly causal linear schemes (one based on two-symbol concatenation in [4] and the other based on one-tap LTI filtering $\left.H(z)=d z^{-1}\right)$ do not outperform the AF scheme, as expected by Theorem 2 .

\section{B. The Multiple-Tap Relay Filter Case: Insights From Ideal Low-Pass Filtering Relays}

In Section IV-A, it is shown that one-tap relay filters do not outperform the AF scheme in flat-fading channels. This is because any one-tap relay filter with a causal or non-causal nonzero delay cannot change the noise spectrum, but destroys the 
coherent signal combining which is available in the AF scheme. However, this is not the case when the relay filter has multiple taps. In this case, the overall noise spectrum as well as the channel gain spectrum in (20) can be shaped by the relay filter, and the LTI relaying scheme with multiple taps can outperform the AF scheme in flat-fading channels. However, the performance analysis in this case is far more difficult than that in the one-tap relay case, especially when the causality constraint is imposed on the relay filter. To circumvent this difficulty, in this subsection we relax the causality constraint on $H(z)$ and consider the tractable ideal low-pass ${ }^{3}$ relay filtering to gain insights into the interaction between the source and relay filters and to assess the rate gain that can be obtained by multiple-tap relay filtering. The frequency response of the ideal low-pass relay filter is given by

$$
H\left(e^{j \omega}\right)= \begin{cases}\delta, & |\omega|<\omega_{c} \\ 0, & \omega_{c}<|\omega|<\pi\end{cases}
$$

where $\delta$ is the passband gain and $\omega_{c}$ is the cutoff frequency. For a given $\omega_{c}$, the optimization problem $(15,17,18)$ with the ideal low-pass relay filter is expressed as

$$
\begin{array}{r}
\max _{\delta, S_{x_{s}}\left(e^{j \omega}\right)}\left[\frac{1}{\pi} \int_{0}^{\omega_{c}} \frac{1}{2} \log _{2}\left(1+\frac{(1+a b \delta)^{2}}{\left(b^{2} \delta^{2}+1\right) \sigma^{2}} S_{x_{s}}\left(e^{j \omega}\right)\right) d \omega\right. \\
\left.+\frac{1}{\pi} \int_{\omega_{c}}^{\pi} \frac{1}{2} \log _{2}\left(1+\frac{1}{\sigma^{2}} S_{x_{s}}\left(e^{j \omega}\right)\right) d \omega\right]
\end{array}
$$

subject to $S_{x_{s}}\left(e^{j \omega}\right) \geq 0, \forall \omega \in[0, \pi]$,

$$
\begin{aligned}
\frac{1}{\pi} \int_{0}^{\pi} S_{x_{s}}\left(e^{j \omega}\right) d \omega-P_{s} & \leq 0 \text { and } \\
\frac{1}{\pi} \int_{0}^{\omega_{c}} \delta^{2}\left(a^{2} S_{x_{s}}\left(e^{j \omega}\right)+\sigma^{2}\right) d \omega-P_{r} & \leq 0
\end{aligned}
$$

where the even symmetry of spectra is used. Note that the problem is not jointly convex w.r.t. $\delta$ and $S_{x_{s}}\left(e^{j \omega}\right)$ for a given $\omega_{c}$. However, we can still apply the Karush-Kuhn-Tucker (KKT) conditions to this problem to obtain the necessary conditions for optimality [24]. The Lagrangian of this problem is given by

$$
\begin{aligned}
\mathcal{L}= & -\frac{1}{\pi} \int_{0}^{\omega_{c}} \frac{1}{2} \log _{2}\left(1+\frac{(1+a b \delta)^{2}}{\left(b^{2} \delta^{2}+1\right) \sigma^{2}} S_{x_{s}}\left(e^{j \omega}\right)\right) d \omega \\
& -\frac{1}{\pi} \int_{\omega_{c}}^{\pi} \frac{1}{2} \log _{2}\left(1+\frac{1}{\sigma^{2}} S_{x_{s}}\left(e^{j \omega}\right)\right) d \omega \\
& +\lambda\left(\frac{1}{\pi} \int_{0}^{\pi} S_{x_{s}}\left(e^{j \omega}\right) d \omega-P_{s}\right) \\
& +\nu\left(\frac{1}{\pi} \int_{0}^{\omega_{c}} \delta^{2}\left(a^{2} S_{x_{s}}\left(e^{j \omega}\right)+\sigma^{2}\right) d \omega-P_{r}\right)
\end{aligned}
$$

where $\lambda$ and $\nu$ are non-negative dual variables. Due to the complementary slackness, either $\lambda=0$ or the source uses full power, i.e., $\frac{1}{\pi} \int_{0}^{\pi} S_{x_{s}}\left(e^{j \omega}\right) d \omega=P_{s}$; and either $\nu=0$ or the relay uses full power. Suppose that the source does not use full power. Then, the source can increase the PSD over the frequency band $\left[\omega_{c}, \pi\right]$ without changing the PSD over $\left[0, \omega_{c}\right)$. Then, the power constraint (37) at the relay is not affected, and

\footnotetext{
${ }^{3}$ Different types of ideal filters, i.e., high-pass, band-pass, band-stop filters, yield essentially the same result as the ideal low-pass filters when the bandwidth of passband is the same.
}

the rate in (35) increases. Thus, the source should use full power to yield maximum rate, and we have $\lambda>0$ due to the complementary slackness. However, the relay may or may not use full power depending on the channel condition. The source PSD solution to the KKT conditions is given by a modified water-filling method:

$S_{x_{s}}\left(e^{j \omega}\right)= \begin{cases}\left(\frac{1}{(2 \ln 2)\left(\lambda+\nu a^{2} \delta^{2}\right)}-\frac{\left(b^{2} \delta^{2}+1\right) \sigma^{2}}{(1+a b \delta)^{2}}\right)^{+}, & |\omega|<\omega_{c}, \\ \left(\frac{1}{(2 \ln 2) \lambda}-\sigma^{2}\right)^{+}, & \omega_{c} \leq|\omega| \leq \pi\end{cases}$

where $\gamma^{+}:=\max \{0, \gamma\}$. The optimal source PSD is given by the difference between the "water level" and the overall effective "noise level." The water level is a function of two dual variables $\lambda$ and $\nu$, and we may have two water levels if $\nu>0$, i.e., the relay also uses full power. The effective noise level $\eta$ is defined as the inverse of the overall CNR density, and it is given by $\eta_{\text {pass }}=\frac{b^{2} \delta^{2}+1}{(1+a b \delta)^{2}} \sigma^{2}$ and $\eta_{\text {stop }}=\sigma^{2}$ for $\left[0, \omega_{c}\right)$ and $\left[\omega_{c}, \pi\right]$, respectively. From here on, we will consider only the case $\eta_{\text {pass }}<$ $\sigma^{2}$. (Otherwise, it is better not to use the relay $(\delta=0)$ since $\sigma^{2}$ over $[0, \pi]$ is the noise level without the relay.) To obtain optimal $\delta$, we need to consider both (35) and (37). By differentiating $\eta_{\text {pass }}(\delta)=\frac{\left(b^{2} \delta^{2}+1\right) \sigma^{2}}{(1+a b \delta)^{2}}$ w.r.t. $\delta$ and setting the derivative to zero, we obtain $\delta=\frac{a}{b}$ for the minimum noise level $\eta_{\text {pass }}^{*}=\frac{\left(1+a^{2}\right) \sigma^{2}}{\left(1+a^{2}\right)^{2}}$. However, we have the relay power constraint (37), yielding $\delta \leq \sqrt{\left.\frac{P_{r}}{\left(\frac{1}{\pi} \int_{0}^{\omega_{c}} a^{2} S_{x_{s}}\left(e^{j \omega}\right) d \omega+\frac{\omega_{c}}{\pi} \sigma^{2}\right.}\right)}=\sqrt{\left.\frac{P_{r}}{\left(a^{2} P_{\mathrm{pass}}\right.}+\left(\frac{\omega_{c}}{\pi}\right) \sigma^{2}\right)}$, where $P_{\text {pass }}$ is the portion of the source power allocated to the relay's passband $\left[0, \omega_{c}\right)$ and $P_{\text {stop }}:=P_{s}-P_{\text {pass }}$. Thus, the optimal $\delta^{*}$ is given by

$$
\delta^{*}=\min \left\{\frac{a}{b}, \sqrt{\frac{P_{r}}{a^{2} P_{\mathrm{pass}}+\frac{\omega_{c}}{\pi} \sigma^{2}}}\right\}
$$

since $\eta_{\text {pass }}(\delta)$ is monotonically increasing as $\delta$ decreases from $\frac{a}{b}$ to zero. With the optimal $\delta^{*}$, the multiple-tap relay filter generates a well in the noise level, as shown in Fig. 3. The effective noise level $\eta_{\text {pass }}(\delta)$ of this well is equal to or lower than that of the AF scheme because the effective noise level for the AF scheme is $\eta_{\mathrm{AF}}(d)=\frac{\left(b^{2} d^{2}+1\right) \sigma^{2}}{(1+a b d)^{2}}$ for $-\pi \leq \omega \leq \pi(\operatorname{see}(22))$ and because for the same source power $P_{s}$ and relay power $P_{r}$ the upper bound for $\delta$ in (40) is larger than that for $d$ in (23). (Note that $P_{\text {pass }} \leq P_{s}$ and $\omega_{c} \leq \pi$.) Thus, when $P_{r}$ is small or $b \ll a$ such that (s.t.) $d^{*}<\frac{a}{b}$ (consequently, $\eta_{\text {pass }}(\delta)<\eta_{\mathrm{AF}}(d)$ ) and when $P_{s}$ is also small enough to be confined within the passband well, the ideal low-pass filtering outperforms the AF scheme. (The amount of gain will be evaluated numerically shortly.) In [4], a modification of AF called the bursty AF is considered. In this scheme, for $0 \leq \tau \leq 1$ fraction of the time the source transmits with power $P_{1}$, and the relay with power $P_{r}$. For the time fraction $\bar{\tau}:=1-\tau$ only the source transmits with power $P_{2}$. To meet the power constraint at the source, we have $\tau P_{1}+\bar{\tau} P_{2}=P_{s}$. The rate of the bursty AF with $P_{\text {pass }}:=\tau P_{1}$ and $P_{\text {stop }}:=\bar{\tau} P_{1}$ coincides with that of the ideal LPF relay filtering. Thus, the ideal LPF relay filtering can be interpreted as the frequency-domain version of the time-domain bursty AF.

The structure of solution $S_{x_{s}}\left(e^{j \omega}\right)$ to the KKT conditions can be classified into four types depending on whether the source power is allocated to the relay's stopband $\left[\omega_{c}, \pi\right]$ or not, and whether the relay uses full power or not (equivalently, $\delta^{*} \neq \frac{a}{b}$ 


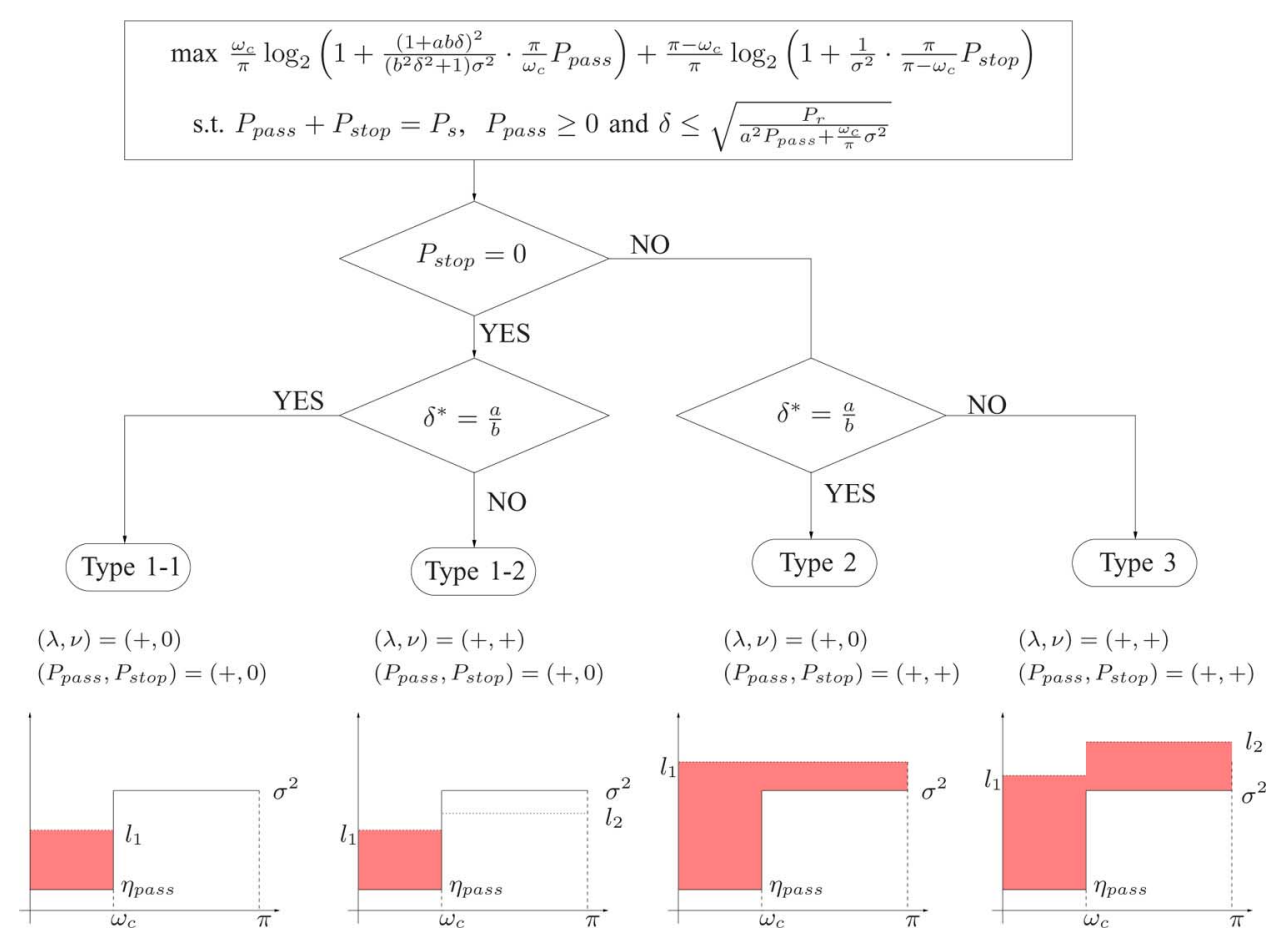

Fig. 3. Different types of the solution to the KKT conditions $\left(l_{1}=\frac{1}{(2 \ln 2)\left(\lambda+\nu a^{2} \delta^{2}\right)}\right.$ and $\left.l_{2}=\frac{1}{(2 \ln 2) \lambda}\right)$.

or not). Fig. 3 shows the solution types. A solution of Type 1 occurs when all source power is allocated to the passband of the relay filter. We can further distinguish Type 1 depending on the power use of the relay. When the relay uses full power, both Lagrange dual variables $\lambda$ and $\nu$ are positive, and there exist two water levels, although no water or power is allocated in the stop band, i.e., $l_{2} \leq \sigma^{2}$ (Type 1-2). Otherwise, we have Type 1-1 in which only $\lambda$ is non-zero. A solution of Type 2 occurs when the relay does not use full power and the source power is allocated over the entire band; there is one water level common to both the passband and stopband. A Type 3 solution occurs when the relay uses full power and the source power is distributed over the entire band; both Lagrange dual variables are positive and the water levels at the passband and stopband are different. Different types of solutions occur for different combinations of parameters, $P_{s}, P_{r}, \sigma^{2}, a, b$, and $\omega_{c}$.

To evaluate the performance of the ideal low-pass filtering at the relay, the optimization problem (35)-(37) was solved numerically using the Matlab function fmincon with $\delta$ and $P_{\text {pass }}$ as variables for given $P_{s}, P_{r}, \sigma^{2}, a, b$ and $\omega_{c}$. First, we fixed $a=1, b=2$ and $\sigma^{2}=1$ (the same as for Fig. 2(a) and (b)), and swept $P_{s}=P_{r}$ for each $\omega_{c} \in\{0.3 \pi, 0.6 \pi\}$, and compared the performance of the ideal low-pass filtering with that of the $\mathrm{AF}$ scheme. The performance is shown in Fig. 4(a) and (b). (The same rate curves are plotted with two different $x$-axis ranges.) It is seen in Fig. 4(a) that indeed there is a gain over the AF scheme at the very low source power values. This is because the low-pass relay filter intentionally generates a well in the noise level in the passband and because all source power is allocated into this well at the very low source power values, as explained already. In this way, the signal is transmitted through a narrowband channel that has a lower noise level than that of the AF scheme. As $P_{s}$ increases, however, the source power spills over the relay's stopband. At high SNR, the effect of the intentional noise level shaping is negligible (the effect of noise itself becomes negligible), and the uniform source power distribution over the entire frequency band is optimal at high SNR. Consequently, filtering out the signal existing at the stopband $\left[\omega_{c}, \pi\right]$ at the relay is detrimental to the performance at high SNR, as shown in Fig. 4(a) and (b). Therefore, we optimized the cutoff frequency $\omega_{c}$ together with $\delta$ and $P_{\text {pass }}$, and the result is shown in Fig. 4(c). It is seen that the optimized solution shows the performance gain over the AF scheme at low SNR and eventually converges to the AF scheme as $P_{s}$ increases. In this case of $a=1$ and $b=2$, the performance gain of the LPF relay over the AF scheme is not significant since the AF scheme performs well already, as shown in Fig. 2(a) and (b) [6, Prop. 9]. Thus, we tried another case of $a=b=2$ and $\sigma^{2}=1$ in which the AF scheme has a noticeable loss from the upper bound, as shown in Fig. 2(c) and (d). Fig. 5 shows the achievable rate in this case. The figure shows similar behavior to the case of $a=1$ and $b=2$. One important fact here is that the performance gain of the ideal low-pass (multiple-tap) relay filtering (even breaking the causality constraint) over the AF scheme is not so significant in both of the cases. Thus, it seems that, practically, there is no need for complicated linear (time-invariant) filtering at the relay beyond the AF scheme in flat-fading channels. However, the insignificant gain is only for flat-fading channels, and this is not the case in realistic ISI channels. In Section V, we will tackle the LTI Gaussian relay problem $(15,17,18,19)$ in ISI channels, propose a practical method to solve this problem, and show that the LTI relay filtering yields a considerable gain over the AF scheme in ISI channels.

\section{Joint Source And Relay Filter Design in ISI CHANNELS}

The LTI relay problem $(15,17,18,19)$ in ISI channels is basically a constrained optimization problem. Our approach to this 


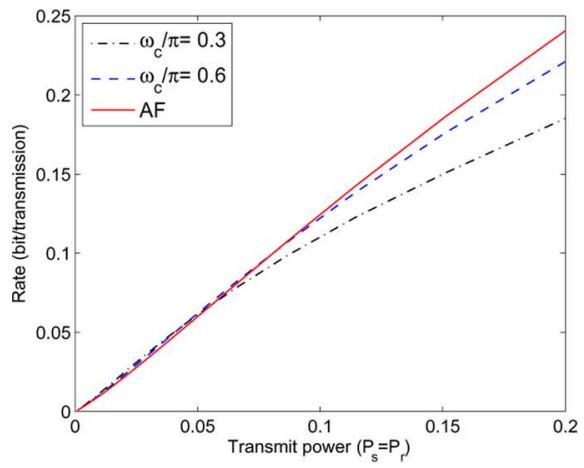

(a)

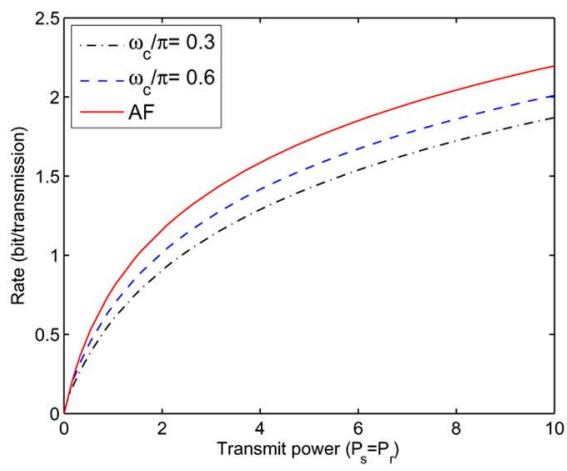

(b)

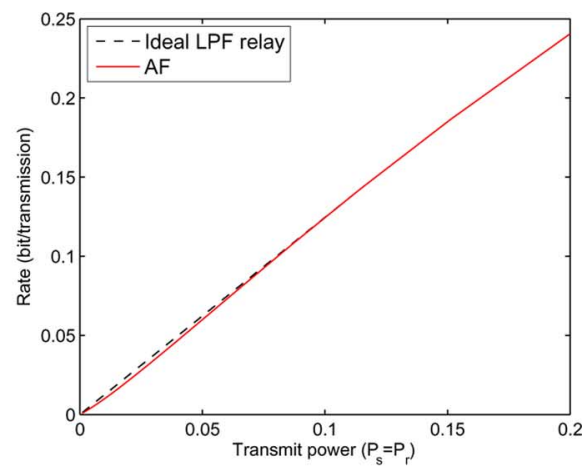

(c)

Fig. 4. Rate performance: Ideal low-pass relay filtering versus $\mathrm{AF}\left(P_{s}=P_{r}, \sigma^{2}=1, a=1\right.$ and $b=2$ ): (a) fixed $\omega_{c}$ (at low $\left.P_{s}\right)$, (b) fixed $\omega_{c}$, and (c) optimized $\omega_{c}$.

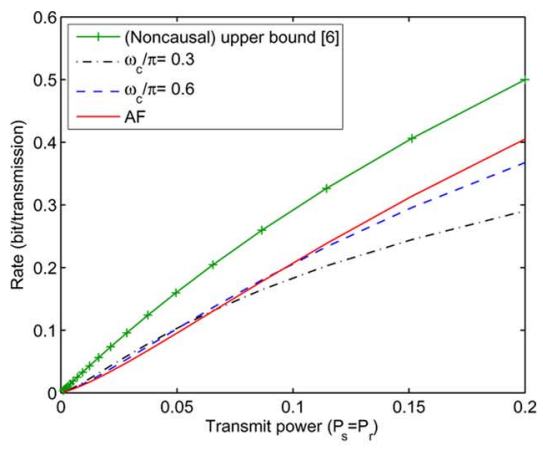

(a)

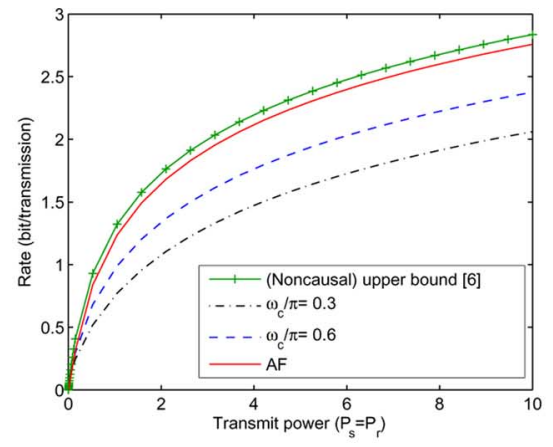

(b)

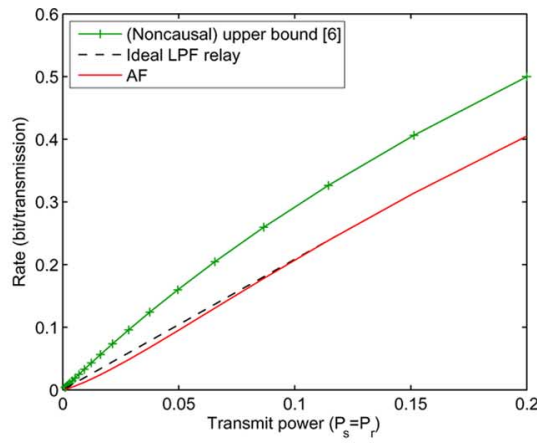

(c)

Fig. 5. Rate performance: Ideal low-pass relay filtering versus AF $\left(P_{s}=P_{r}, \sigma^{2}=1, a=2\right.$ and $b=2$ ): (a) fixed $\omega_{c}$ (at low $\left.P_{s}\right)$, (b) fixed $\omega_{c}$, and (c) optimized $\omega_{c}$.

problem is based on a powerful tool of the (adaptive) projected (sub)gradient method developed by Goldstein [18], Polyak [19] and Yamada et al. [20], [21]. In Section V-A, we will briefly introduce their results that are relevant to our problem, and then move on to the original LTI relay problem.

\section{A. Background: The Adaptive Projected Subgradient Method (APSM) [20], [21]}

The projected gradient and subgradient methods were initially proposed by Goldstein [18] and Polyak [19], respectively, to solve cost minimization problems over convex constraint sets. Recently, Yamada et al. fully generalized the methods even to adaptive situations with time-varying cost functions, and proved strong convergence of the methods [20], [21].

Definition 2 (Subdifferential): Let $\phi: \mathcal{H} \rightarrow \mathbb{R}$ be a continuous convex function from a Hilbert space $\mathcal{H}$ to the set $\mathbb{R}$ of real numbers. Then, the subdifferential $\partial \phi(\mathbf{u})$ of $\phi$ at $\mathbf{u}$ is defined as the set of all the subgradients of $\phi$ at $\mathbf{u}$, i.e., $\partial \phi(\mathbf{u}):=\{\mathbf{g} \in$ $\mathcal{H} \mid<\mathbf{v}-\mathbf{u}, \mathbf{g}>+\phi(\mathbf{u}) \leq \phi(\mathbf{v}), \forall \mathbf{v} \in \mathcal{H}\}$, where $<\cdot, \cdot>$ is the inner product between two vectors.

Here, $\langle\mathbf{v}-\mathbf{u}, \mathbf{g}\rangle+\phi(\mathbf{u})=r$ is a hyperplane with coordinates $(\mathbf{v}, r)$ passing $(\mathbf{u}, \phi(\mathbf{u}))$ in the space $\mathcal{H} \times \mathbb{R}$. Thus, the condition defining the subgradient implies that the hyperplane $<\mathbf{v}-\mathbf{u}, \mathbf{g}>+\phi(\mathbf{u})=r$ is below the cost surface $\phi(\mathbf{v})=r$. It is known that $\partial \phi(\mathbf{u})$ is nonempty, and by definition we have $\mathbf{0} \in \partial \phi(\mathbf{u}) \Leftrightarrow \phi(\mathbf{u})=\min _{\mathbf{v} \in \mathcal{H}} \phi(\mathbf{v})$. In the differentiable case, the gradient is a unique subgradient.
Definition 3 (Metric Projection): The metric projection $P_{K}(\mathbf{u})$ of a point $\mathbf{u}$ onto a closed convex set $K$ is the closest point in $K$ from $\mathbf{u}$, i.e., $\left\|P_{K}(\mathbf{u})-\mathbf{u}\right\| \leq\|\mathbf{v}-\mathbf{u}\|, \forall \mathbf{v} \in K$.

Definition 4 (Subgradient Projection): Suppose that a continuous convex function $\phi: \mathcal{H} \rightarrow \mathbb{R}$ satisfies $\operatorname{lev}_{\leq 0} \phi:=\{\mathbf{v} \in$ $\mathcal{H} \mid \phi(\mathbf{v}) \leq 0\} \neq \emptyset$, i.e., the zero-level set is not empty. Let $\phi^{\prime}: \mathcal{H} \rightarrow \mathcal{H}$ be a selection of the subdifferential $\partial \phi$, i.e., $\phi^{\prime}(\mathbf{u}) \in \partial \phi(\mathbf{u}), \forall \mathbf{u} \in \mathcal{H}$. Then, a mapping $T_{s p(\phi)}: \mathcal{H} \rightarrow \mathcal{H}$ defined by

$$
T_{s p(\phi)}: \mathbf{u} \longmapsto \begin{cases}\mathbf{u}-\frac{\phi(\mathbf{u})}{\left\|\phi^{\prime}(\mathbf{u})\right\|^{2}} \phi^{\prime}(\mathbf{u}), & \text { if } \phi(\mathbf{u})>0 \\ \mathbf{u}, & \text { if } \phi(\mathbf{u}) \leq 0\end{cases}
$$

is called a subgradient projection relative to $\phi$.

Here, the normalization of the subgradient by factor $\frac{\phi(\mathbf{u})}{\left\|\phi^{\prime}(\mathbf{u})\right\|^{2}}$ makes it easy to determine the step size parameter for adaptive algorithm construction. Basically, the subgradient projection moves the current point $\mathbf{u}$ to its metric projection onto the intersection of two hyperplanes $r=0$ and $\langle\mathbf{v}-\mathbf{u}, \mathbf{g}\rangle$ $+\phi(\mathbf{u})=0$ in the space of $\mathcal{H} \times \mathbb{R}$ with coordinates $(\mathbf{v}, r)$. (See [25, Fig. 16].) It is known that the subgradient projection always moves the original point closer to every point in the zero-level set, which is known as the Fejér monotone property with respect to the zero-level set [21], [26]. It is also known that the subgradient projection belongs to the class of firmly quasi-nonexpansive mappings [21], [26]. Now, we state the adaptive projected subgradient theorem. 
Theorem 4 (APSM [20], [21]): Let $\phi_{n}: \mathcal{H} \rightarrow[0, \infty)(\forall n \in$ $\mathbb{N}$ ) be a sequence of continuous convex functions and $K \subset \mathcal{H}$ a nonempty closed convex set. For an arbitrarily given $\mathbf{u}_{0} \in K$, the sequence $\left\{\mathbf{u}_{n}\right\}_{n \in \mathbb{N}} \subset K$ generated by the adaptive projected subgradient method:

$$
\mathbf{u}_{n+1}= \begin{cases}P_{K}\left(\mathbf{u}_{n}-\mu_{n} \frac{\phi_{n}\left(\mathbf{u}_{n}\right)}{\left\|\phi_{n}^{\prime}\left(\mathbf{u}_{n}\right)\right\|^{2}} \phi_{n}^{\prime}\left(\mathbf{u}_{n}\right)\right), & \text { if } \phi_{n}^{\prime}\left(\mathbf{u}_{n}\right) \neq 0 \\ \mathbf{u}_{n}, & \text { otherwise }\end{cases}
$$

where $\phi_{n}^{\prime}\left(\mathbf{u}_{n}\right) \in \partial \phi_{n}\left(\mathbf{u}_{n}\right)$ and $0 \leq \mu_{n} \leq 2$, satisfies the following.

- (Monotone approximation) Suppose

$$
\mathbf{u}_{n} \notin \Omega_{n}:=\left\{\mathbf{u} \in K \mid \phi_{n}(\mathbf{u})=\phi_{n}^{*}:=\inf _{\mathbf{v} \in K} \phi_{n}(\mathbf{v})\right\} \neq \emptyset
$$

Then, by using $\forall \mu_{n} \in\left(0,2\left(1-\frac{\phi_{n}^{*}}{\phi_{n}\left(\mathbf{u}_{n}\right)}\right)\right)$, we have $\left\|\mathbf{u}_{n+1}-\mathbf{u}_{(n)}^{*}\right\|<\left\|\mathbf{u}_{n}-\mathbf{u}_{(n)}^{*}\right\|$ for all $\mathbf{u}_{(n)} \in \Omega_{n}$.

- (Asymptotic optimality) Suppose

$\exists N_{0} \in \mathbb{N}$ s.t. $\phi_{n}^{*}=0 \forall n \geq N_{0}$ and $\Omega:=\cap_{n \geq N_{0}} \Omega_{n} \neq \emptyset$.

Then, $\left\{\mathbf{u}_{n}\right\}_{n \in \mathbb{N}}$ is bounded. Moreover, if we specially use $\forall \mu_{n} \in\left[\epsilon_{1}, 2-\epsilon_{2}\right] \subset(0,2)$, we have $\lim _{n \rightarrow \infty} \phi_{n}\left(\mathbf{u}_{n}\right)=0$ provided that $\left\{\phi_{n}^{\prime}\left(\mathbf{u}_{n}\right)\right\}_{n \in \mathbb{N}}$ is bounded.

- (Strong convergence) Assume (44) and $\Omega$ has some relative interior w.r.t. a hyperplane $\Pi(\subset \mathcal{H})$; i.e., there exist $\tilde{\mathbf{u}} \in \Pi \cap \Omega$ and $\exists \epsilon>0$ satisfying $\{\mathbf{u} \in \Pi$ s.t. $\|\mathbf{u}-\tilde{\mathbf{u}}\| \leq \epsilon\} \subset \Omega$. Then, by using $\forall \eta_{n} \in\left[\epsilon_{1}, 2-\epsilon_{2}\right] \subset(0,2),\left\{\mathbf{u}_{n}\right\}_{n \in \mathbb{N}}$ converges strongly to some $\hat{\mathbf{u}} \in K$, i.e., $\lim _{n \rightarrow \infty}\left\|\mathbf{u}_{n}-\hat{\mathbf{u}}\right\|=0$. Moreover, $\lim _{n \rightarrow \infty} \phi_{n}(\hat{\mathbf{u}})=0$ if $\left\{\phi_{n}^{\prime}\left(\mathbf{u}_{n}\right)\right\}_{n \in \mathbb{N}}$ is bounded and if there exists bounded $\left\{\phi_{n}^{\prime}(\hat{\mathbf{u}})\right\}_{n \in \mathbb{N}}$, where $\phi_{n}^{\prime}(\hat{\mathbf{u}}) \in \partial \phi_{n}(\hat{\mathbf{u}}), \forall n \in \mathbb{N}$.

Note that the update rule in (42) is a composite projection composed of the subgradient based projection relative to $\phi_{n}$ and the metric projection onto $K$. In fact, the first projection is not the subgradient projection exactly since the step size parameter $\mu_{n}$ does not need to be one. This projection can be rewritten as

$$
\begin{aligned}
\hat{T}_{\mu_{n}}\left(\mathbf{u}_{n}\right): & =\mathbf{u}_{n}-\mu_{n} \frac{\phi_{n}\left(\mathbf{u}_{n}\right)}{\left\|\phi_{n}^{\prime}\left(\mathbf{u}_{n}\right)\right\|^{2}} \phi_{n}^{\prime}\left(\mathbf{u}_{n}\right) \\
& =\left[\left(1-\mu_{n}\right) I+\mu_{n} T_{s p\left(\phi_{n}\right)}\right]\left(\mathbf{u}_{n}\right)
\end{aligned}
$$

where $I$ is the identity mapping. When $\mu_{n}=1, \hat{T}_{\mu_{n}}=T_{s p\left(\phi_{n}\right)}$. When $\mu_{n}<1$, the projection moves the original point towards the exact subgradient projection point, but not fully. When $\mu_{n}>$ 1 , on the other hand, the projection moves the original point beyond the exact subgradient projection point. For any $\mu_{n} \in$ $(0,2), \hat{T}_{\mu_{n}}$ is called a $\mu_{n}$-averaged quasi-nonexpansive mapping, and the properties of this mapping play an important role in the proof of Theorem 4 . The difference of the projected subgradient method from the projected gradient method [18] without the additional normalization factor $\frac{\phi_{n}\left(\mathbf{u}_{n}\right)}{\left\|\phi_{n}^{\prime}\left(\mathbf{u}_{n}\right)\right\|^{2}}$ is that we know the exact range of the step size parameter $\mu_{n}$ for convergence in the case of the projected subgradient method. Furthermore, the projected gradient method [18] with sufficiently small positive step size $\kappa_{n}$ s.t. $\kappa_{n}:=\mu_{n} \frac{\phi_{n}\left(\mathbf{u}_{n}\right)}{\left\|\phi_{n}^{\prime}\left(\mathbf{u}_{n}\right)\right\|^{2}}<\frac{\phi_{n}\left(\mathbf{u}_{n}\right)}{\left\|\phi_{n}^{\prime}\left(\mathbf{u}_{n}\right)\right\|^{2}}, \forall n$, is a special case of the APSM since the projection (45) used to update the variable in (42) does not need to be the exact subgradient projection. The above result is general and can be applied to many constrained optimization problems. Consider the simple case with a fixed cost function (i.e., $\phi_{n}(\mathbf{u}) \equiv \phi(\mathbf{u}) \forall n$ ) of which minimum is achieved in $K$ and known. Let $\phi^{*}=$ $\min _{\mathbf{u} \in K} \phi(\mathbf{u})$. Then, we can define $\tilde{\phi}(\mathbf{u}):=\phi(\mathbf{u})-\phi^{*}$. Then, the condition (44) is trivially satisfied by $\tilde{\phi}(\mathbf{u})$, and we have $\tilde{\phi}^{\prime}(\mathbf{u})=\phi^{\prime}(\mathbf{u})$ and $\lim _{n \rightarrow \infty} \tilde{\phi}\left(\mathbf{u}_{n}\right)=0$, i.e., $\lim _{n \rightarrow \infty} \phi\left(\mathbf{u}_{n}\right)=$ $\phi^{*}$. In this case, the result reduces to the Polyak's projected subgradient method for which he showed the weak convergence [19, Theorem 1]. (For the proof of Theorem 4, refer to [21]. For the introduction of related projections, see [25].)

\section{B. Joint Source and Relay Filter Design Via the Adaptive Projected Subgradient Method}

Even under the LTI formulation $(15,17,18,19)$, the search space for source and relay filters $T(z)$ and $H(z)$ has countably-infinite dimensions since both $T(z)$ and $H(z)$ can be infinite impulse response (IIR) filters. To avoid the difficulties in searching in an infinite dimensional space and in imposing the stability condition, we restrict ourselves to the case that both $T(z)$ and $H(z)$ have FIRs as in most practical filtering applications. Then, the stability and causality constraints are automatically satisfied. In this case, the source and relay filter responses are respectively given by

$$
\begin{aligned}
& T(z)=t_{0}+t_{1} z^{-1}+\cdots+t_{L_{s}-1} z^{-L_{s}+1} \text { and } \\
& H(z)=h_{0}+h_{1} z^{-1}+\cdots+h_{L_{r}-1} z^{-L_{r}+1}
\end{aligned}
$$

where $L_{s}$ and $L_{r}$ are the orders of source and relay filters, respectively. Although the channel responses $H_{\mathrm{sr}}(z), H_{\mathrm{rd}}(z)$ and $H_{\mathrm{sd}}(z)$ do not need to have finite durations, we also assume that these channel responses have finite duration $L$ for simplicity. For notational convenience, we define the following:

$$
\begin{aligned}
\mathbf{w}_{m}(\omega) & :=\left[1, e^{j \omega}, e^{j 2 \omega}, \ldots, e^{j(m-1) \omega}\right]^{T} \\
\mathbf{t} & :=\left[t_{0}, t_{1}, \ldots, t_{L_{s}-1}\right]^{T} \in \mathbb{R}^{L_{s} \times 1} \\
\mathbf{h} & :=\left[h_{0}, h_{1}, \ldots, h_{L_{r}-1}\right]^{T} \in \mathbb{R}^{L_{r} \times 1} \\
\mathbf{u} & :=\left[\mathbf{t}^{T}, \mathbf{h}^{T}\right]^{T} \in \mathbb{R}^{\left(L_{s}+L_{r}\right) \times 1} \\
\mathbf{h}_{\mathrm{ab}} & :=\left[h_{\mathrm{ab}}[0], h_{\mathrm{ab}}[1], \ldots, h_{\mathrm{ab}}[L-1]\right]^{T} \text { for } \\
(a, b) & =(s, r),(r, d) \text { and }(s, d) .
\end{aligned}
$$

Then, we have $T\left(e^{j \omega}\right)=\mathbf{w}_{L_{s}}^{H}(\omega) \mathbf{t}, H\left(e^{j \omega}\right)=\mathbf{w}_{L_{r}}^{H}(\omega) \mathbf{h}$, and $H_{\mathrm{ab}}\left(e^{j \omega}\right)=\mathbf{w}_{L}^{H}(\omega) \mathbf{h}_{\mathrm{ab}}$. Here, we assume that $\mathbf{t}$ and $\mathbf{h}$ are real vectors for simplicity, but the extension to the complex case is straightforward. Now from (15) we define the cost function as (53) at the bottom of the page, where the respective dependence of $H\left(e^{j \omega}\right)$ and $T\left(e^{j \omega}\right)$ on $\mathbf{h}$ and $\mathbf{t}$ is explicitly shown. Note here that the cost function $\phi(\cdot)(\leq 0 \forall \mathbf{u})$ is time-invariant since the

$$
\phi(\mathbf{u})=-\frac{1}{2 \pi} \int_{-\pi}^{\pi} \frac{1}{2} \log _{2}\left(1+\frac{\left|H_{\mathrm{sd}}\left(e^{j \omega}\right)+H_{\mathrm{sr}}\left(e^{j \omega}\right) H\left(e^{j \omega} ; \mathbf{h}\right) H_{\mathrm{rd}}\left(e^{j \omega}\right)\right|^{2}}{\sigma^{2}\left(\left|H_{\mathrm{rd}}\left(e^{j \omega}\right) H\left(e^{j \omega} ; \mathbf{h}\right)\right|^{2}+1\right)}\left|T\left(e^{j \omega} ; \mathbf{t}\right)\right|^{2}\right) d \omega,
$$


channels are static. The gradient $\phi^{\prime}(\mathbf{u})$ at $\mathbf{u}$ can be obtained after some computation as

$$
\begin{aligned}
\phi^{\prime}(\mathbf{u}) & =\frac{1}{2 \pi} \int_{-\pi}^{\pi} \frac{1}{2 \ln 2} \cdot \frac{1}{1+A(\omega)} \\
& \times\left(\begin{array}{c}
B(\omega) \\
C_{1}(\omega)\left(C_{2}(\omega)-C_{3}(\omega) C_{4}(\omega)\right)
\end{array}\right) d \omega
\end{aligned}
$$

where $A(\omega)=\operatorname{CNR}\left(e^{j \omega} ; \mathbf{h}\right) \cdot \mathbf{t}^{T} \mathbf{w}_{L_{s}} \mathbf{w}_{L_{s}}^{H} \mathbf{t}, B(\omega)=$ $\operatorname{CNR}\left(e^{j \omega} ; \mathbf{h}\right) \cdot\left(\mathbf{w}_{L_{s}} \mathbf{w}_{L_{s}}^{H}+\mathbf{w}_{L_{s}}^{*} \mathbf{w}_{L_{s}}^{T}\right) \mathbf{t}, C_{1}(\omega)=\frac{\mathbf{t}^{T} \mathbf{w}_{L_{s}} \mathbf{w}_{L_{s}}^{H} \mathbf{t}}{D\left(e^{j \omega} ; \mathbf{h}\right)}$, $C_{2}(\omega)=\nabla_{\mathbf{h}} N\left(e^{j \omega} ; \mathbf{h}\right), C_{3}(\omega)=\nabla_{\mathbf{h}} D\left(e^{j \omega} ; \mathbf{h}\right)$ and $C_{4}(\omega)=\operatorname{CNR}\left(e^{j \omega} ; \mathbf{h}\right)$; and $N\left(e^{j \omega} ; \mathbf{h}\right)$ and $D\left(e^{j \omega} ; \mathbf{h}\right)$ are the numerator and denominator of $\operatorname{CNR}\left(e^{j \omega} ; \mathbf{h}\right)$, respectively, defined in (16). The source power constraint (17) is given in terms of $\mathbf{t}$ and $\mathbf{h}$ by

$$
\frac{1}{2 \pi} \int_{-\pi}^{\pi}\left|T\left(e^{j \omega}\right)\right|^{2} d \omega=\sum_{l=0}^{L_{s}-1} t_{l}^{2}=\|\mathbf{t}\|^{2} \leq P_{s}
$$

by Parseval's theorem, and the constraint set for $\mathbf{t}$ determined by (55) is simply a ball in $\mathbf{t}$, denoted by $B_{\mathbf{t}}\left(P_{s}\right)$, with no constraint on $\mathbf{h}$. Next, consider the power constraint at the relay. The relay power constraint (18) is expressed in terms of $\mathbf{t}$ and $\mathbf{h}$ as

$$
\begin{aligned}
\frac{1}{2 \pi} & \int_{-\pi}^{\pi}\left|H\left(e^{j \omega}\right)\right|^{2}\left(\left|H_{\mathrm{sr}}\left(e^{j \omega}\right)\right|^{2} \cdot\left|T\left(e^{j \omega}\right)\right|^{2}+\sigma^{2}\right) d \omega \\
= & \frac{1}{2 \pi} \int_{-\pi}^{\pi} \mathbf{h}^{T} \mathbf{w}_{L_{r}}(\omega) \mathbf{w}_{L_{r}}^{H}(\omega) \mathbf{h} \\
& \cdot \underbrace{\left(\left|H_{\mathrm{sr}}\left(e^{j \omega}\right)\right|^{2} \mathbf{t}^{T} \mathbf{w}_{L_{s}}(\omega) \mathbf{w}_{L_{s}}^{H}(\omega) \mathbf{t}+\sigma^{2}\right)}_{=: g(\omega ; \mathbf{t})} d \omega, \\
= & \mathbf{h}^{T}\left(\frac{1}{2 \pi} \int_{-\pi}^{\pi} g(\omega ; \mathbf{t}) \mathbf{w}_{L_{r}}(\omega) \mathbf{w}_{L_{r}}^{H}(\omega) d \omega\right) \mathbf{h} \leq P_{r} .
\end{aligned}
$$

Note that the constraint set for $(\mathbf{t}, \mathbf{h})$ determined by (56) is bi-convex, i.e., convex in each of $\mathbf{t}$ and $\mathbf{h}$ but not jointly convex, as seen in (57). The constraint set for $\mathbf{h}$ for a given $\mathbf{t}$ is an ellipsoid, as seen in (58). Let us denote this ellipsoid by $\xi_{\mathbf{h}}(\mathbf{t})$. The above inequality can also be written as

$$
\begin{array}{r}
\mathbf{t}^{T}\left(\frac{1}{2 \pi} \int_{-\pi}^{\pi}\left(\left|H_{\mathrm{sr}}\left(e^{j \omega}\right)\right|^{2} \mathbf{h}^{T} \mathbf{w}_{L_{r}}(\omega) \mathbf{w}_{L_{r}}^{H}(\omega) \mathbf{h}\right) \mathbf{w}_{L_{s}}(\omega) \mathbf{w}_{L_{s}}^{H}(\omega) d \omega\right) \mathbf{t} \\
+\frac{\sigma^{2}}{2 \pi} \int_{-\pi}^{\pi} \mathbf{h}^{T} \mathbf{w}_{L_{r}}(\omega) \mathbf{w}_{L_{r}}^{H}(\omega) \mathbf{h} d \omega \leq P_{r}
\end{array}
$$

and, therefore, the constraint set for $\mathbf{t}$ for a given $\mathbf{h}$ is also an ellipsoid.

Now, the rate maximization problem under the LTI FIR formulation is given by

$$
\min _{\mathbf{t}, \mathbf{h}} \phi(\mathbf{t}, \mathbf{h})
$$

such that

$$
(\mathbf{t}, \mathbf{h}) \in C_{1} \text { and }(\mathbf{t}, \mathbf{h}) \in C_{2}
$$

where $\phi(\mathbf{t}, \mathbf{h})$ is given by (53), and $C_{1}$ and $C_{2}$ are the constraint sets determined by (55) and (58), respectively. The situation is depicted in Fig. 6. Even though $\phi(\mathbf{t}, \mathbf{h})$ is not jointly convex in $\mathbf{t}$ and $\mathbf{h}$, we can still apply the projected (sub)gradient method

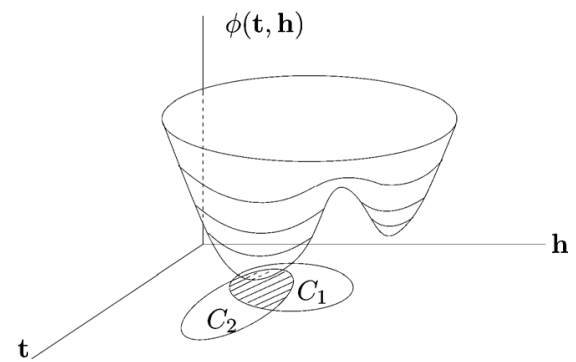

Fig. 6. Cost minimization over the intersection of two constraint sets.

in the previous subsection. (Since we do not know the minimum $\phi^{*}$ of $\phi(\mathbf{u})$, we cannot explicitly construct $\tilde{\phi}(\mathbf{u})=\phi(\mathbf{u})-\phi^{*}$ to satisfy the conditions in Theorem 4; the zero-level set of the cost function should not be empty. Thus, to eliminate the necessity of the knowledge of $\phi^{*}$, we use sufficiently small ${ }^{4}$ $\kappa_{n}\left(=\frac{\mu_{n} \tilde{\phi}\left(\mathbf{u}_{n}\right)}{\left\|\tilde{\phi}^{\prime}\left(\mathbf{u}_{n}\right)\right\|^{2}}\right)$ directly. That is, we use the projected gradient version of the APSM.) Suppose now that $K:=C_{1} \cap C_{2}$ is convex. Then, applying the projected (sub)gradient method with $\mathbf{u}_{0} \in K$ leads to the convergence to a local minimum at least. Due to the nonconvexity of $C_{2}$, however, $K$ is not a convex set. To circumvent this issue, let us investigate the structure of the two constraint sets further. Fig. 7 shows the two constraint sets, $C_{1}$ and $C_{2}$, and their intersection $K$ in the case of $\mathbf{t}=\left(t_{0}, t_{1}\right)$ and $\mathbf{h}=h_{0} . C_{1}$ and $C_{2}$ are the red cylinder and the blue shape that looks like a mountain on one side and is symmetric about the $h_{0}=0$ plane in the left side of Fig. 7, respectively. In this case, the ellipsoid $\xi_{\mathbf{h}}\left(\mathbf{t}^{\prime}\right)$ is a line segment passing through $\left(\mathbf{t}^{\prime}, 0\right)$ ending at the upper and the lower surfaces of the blue shape, as shown in Fig. 7. As mentioned already, for a given $\mathbf{h}$ the constraint set for $\mathbf{t}$ is an ellipsoid. (See (59).) So, we have an ellipsoidal cut of the blue shape by a plane perpendicular to the $h_{0}$-axis. Thus, $C_{1} \cap C_{2}$ is given by the shape in the right side of Fig. 7, which looks like a cylinder with bulges on the top and the bottom. If we are willing to sacrifice the bulging regions from the feasibility set, we can construct a convex set $\tilde{K} \subset K$ as the Cartesian product of $B_{\mathbf{t}}\left(P_{s}\right)$ and $\Xi_{\mathbf{h}}\left(P_{s}, P_{r}\right)$, i.e.,

$$
\tilde{K}:=B_{\mathbf{t}}\left(P_{s}\right) \times \Xi_{\mathbf{h}}\left(P_{s}, P_{r}\right)
$$

where

$$
\Xi_{\mathbf{h}}\left(P_{s}, P_{r}\right)=\bigcap_{\mathbf{t} \in B_{\mathbf{t}}\left(P_{s}\right)} \xi_{\mathbf{h}}(\mathbf{t})=\bigcap_{\mathbf{t} \in \operatorname{surface}\left(B_{\mathbf{t}}\left(P_{s}\right)\right)} \xi_{\mathbf{h}}(\mathbf{t}) .
$$

The second equality in (63) is because $\xi_{\mathbf{h}}\left(\mathbf{t}^{\prime}\right) \subset \xi_{\mathbf{h}}\left(\gamma \mathbf{t}^{\prime}\right)$ for $\gamma \in$ $[0,1]$. This can easily be verified from (56). For $\gamma \mathbf{t}^{\prime},\left|T\left(e^{j \omega}\right)\right|^{2}$ will simply scale down to $\gamma^{2}\left|T\left(e^{j \omega}\right)\right|^{2}$, and $\left|H\left(e^{j \omega}\right)\right|^{2}$ satisfying the inequality with $\left|T\left(e^{j \omega}\right)\right|^{2}$ will satisfy the inequality with $\gamma^{2}\left|T\left(e^{j \omega}\right)\right|^{2}$. Here, $\Xi_{\mathbf{h}}\left(P_{s}, P_{r}\right)$ is convex since it is the intersection of ellipsoids. Also, $\tilde{K}$ is convex due to its Cartesian product structure. In the considered case of $\left(t_{0}, t_{1}, h_{0}\right), \tilde{K}$ is exactly the red cylinder in the right side of Fig. 7. Based on the above discussion, we now provide our first algorithm for the joint source and relay filter design.

\footnotetext{
${ }^{4}$ In the case of the projected gradient method, it is known that the algorithm converges to the true minimum for a convex problem if $\kappa_{n} \in\left(0, \frac{2}{\zeta}\right)$ for $\zeta$-Lipschitzian $\phi^{\prime}(\cdot)$ [18], [27].
} 


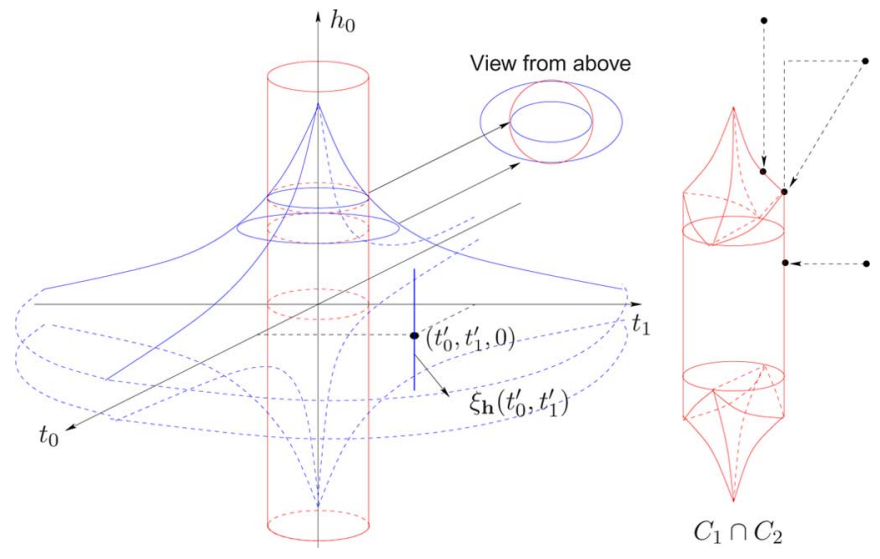

Fig. 7. The structure of two constraint sets in case of $\mathbf{t}=\left(t_{0}, t_{1}\right)$ and $\mathbf{h}=h_{0}$.

\section{Algorithm 1:}

- Initialization: Set $\mathbf{u}_{0}$ properly, e.g.,

$$
\mathbf{u}_{0}=\left[\mathbf{t}_{0}^{T}, \mathbf{0}^{T}\right]^{T}
$$

with $\mathbf{t}_{0}=\left[\sqrt{\frac{P_{s}}{L_{s}}}[1,1, \ldots, 1]^{T}\right.$. (It is easy to verify that this $\mathbf{u}_{0} \in \tilde{K} \subset K$.)

- Update:

$\mathbf{u}_{n+1}= \begin{cases}P_{\tilde{K}}\left(\mathbf{u}_{n}-\kappa_{n} \phi^{\prime}\left(\mathbf{u}_{n}\right)\right), & \text { if } \phi^{\prime}\left(\mathbf{u}_{n}\right) \neq 0 \\ \mathbf{u}_{n}, & \text { otherwise, }\end{cases}$

for some $\left\{\kappa_{n}>0\right\}$, where $\phi^{\prime}(\mathbf{u})$ is given by (54), and $\mathbf{u}_{n}=\left[\mathbf{t}_{n}^{T}, \mathbf{h}_{n}^{T}\right]^{T}$.

- Stopping rule: Stop the update either when $\mathbf{u}_{n}$ does not change further or when the number of iterations exceeds a preset maximum.

Due to the Cartesian product structure of $\tilde{K}$ in (62), the metric projection $P_{\tilde{K}}$ can be implemented by the composition of two separate projections, one in $\mathbf{t}$ projecting the $\mathbf{t}$-coordinates onto $B_{\mathbf{t}}\left(P_{s}\right)$ and then the other in $\mathbf{h}$ projecting the $\mathbf{h}$-coordinates onto $\Xi_{\mathbf{h}}\left(P_{s}, P_{r}\right)$, i.e.,

$$
P_{\tilde{K}}=P_{\Xi_{\mathbf{h}}\left(P_{s}, P_{r}\right)} \circ P_{B_{\mathbf{t}}\left(P_{s}\right)}
$$

where the projection onto a ball is simply given by

$$
P_{B_{\mathbf{t}}\left(P_{s}\right)}(\mathbf{t})= \begin{cases}\mathbf{t}, & \text { if }\|\mathbf{t}\|^{2} \leq P_{s} \\ \frac{\sqrt{P_{s}} \mathbf{t}}{\|\mathbf{t}\|,} & \text { otherwise }\end{cases}
$$

and $P_{\Xi_{\mathbf{h}}\left(P_{s}, P_{r}\right)}$ can be approximated by using the successive projection method [26]. That is, we uniformly partition the surface of the $L_{s}$-dimensional ball with radius $\sqrt{P_{s}}$ into $M$ subsets, and select the center $\mathbf{t}_{m}$ of subset $m$. Then, $\Xi_{\mathbf{h}}\left(P_{s}, P_{t}\right) \approx$ $\cap_{m=1}^{M} \xi_{\mathbf{h}}\left(\mathbf{t}_{m}\right)$ and

$$
P_{\Xi_{\mathbf{h}}\left(P_{s}, P_{r}\right)}(\mathbf{h}) \approx P_{\xi_{\mathbf{h}}\left(\mathbf{t}_{M}\right)} \circ P_{\xi_{\mathbf{h}}\left(\mathbf{t}_{M-1}\right)} \circ \cdots \circ P_{\xi_{\mathbf{h}}\left(\mathbf{t}_{1}\right)}(\mathbf{h})
$$

where the projection onto an ellipsoid can easily be implemented by a known method like the one in [28]. If $\phi^{\prime}(\mathbf{u}) \neq 0$ for all $\mathbf{u} \in \tilde{K}$, then Algorithm 1 will find the point that yields the maximum rate within $\tilde{K}$. Otherwise, Algorithm 1 finds a local optimum attracting $\mathbf{u}_{0}$, and convergence is guaranteed. However, the complexity of the projection $P_{\Xi_{\mathbf{h}}\left(P_{s}, P_{r}\right)}(\mathbf{h})$ is prohibitive even for small values of $L_{s}$. Thus, we propose a simplified algorithm to eliminate this difficulty in the following.

Algorithm 2:

- Initialization of $\mathbf{u}_{0}$.

- Update: By (69) at the bottom of the page.

- Stopping rule: The same as that in Algorithm 1.

In the initialization step, we can consider other initial points than the example in Algorithm 1. For example,

$$
\mathbf{t}_{0}=\left[\sqrt{P_{s}}, 0, \ldots, 0\right]^{T} \text { and } \mathbf{h}_{0}=P_{\xi_{\mathbf{h}}\left(\mathbf{t}_{0}\right)}\left([1, \ldots, 1]^{T}\right) .
$$

With such an initial point, we can start the algorithm from the full power use at the relay. In Algorithm 2, the gradient descent is the same as that in Algorithm 1, but the projection to $K$ is now different. Here, we first project only the $\mathbf{t}_{n}$ coordinates to the ball $B_{\mathbf{t}}\left(P_{s}\right)$, and then project the $\mathbf{h}_{n}$ coordinates onto the h-ellipsoid $\xi_{\mathbf{h}}\left(P_{B_{\mathbf{t}}\left(P_{s}\right)}\left(\mathbf{t}_{n}\right)\right)$ corresponding to $P_{B_{\mathbf{t}}\left(P_{s}\right)}\left(\mathbf{t}_{n}\right)$ given by (58); several projection examples in the case of $L_{s}=2$ and $L_{r}=1$ are shown in the right side of Fig. 7. In this way, the metric projection to $K$ is approximated and highly simplified, and $\mathbf{u}_{n} \in K$ for all $n$. The convergence of Algorithm 2 is not guaranteed theoretically, but it is observed numerically that Algorithm 2 is stable and works well. This is because $K$ seems almost convex as seen in the example in Fig. 7 and the proposed two-step projection onto $K$ approximates the metric projection onto $K$ well due to the almost cylindrical structure of $K$ except the top and bottom. Under our formulation, it is straightforward to impose the strict causality constraint on the relay filter $H(z)$ that captures the possible delay at the relay for digital processing; simply remove $h_{0}$ in (47) and follow the same procedure as the causal case.

Up to here, the algorithms have been developed for static linear relay channels. However, the capability of handling timevarying cost functions by the APSM enables us to apply the proposed filter design algorithms to the time-varying channel case beyond the static channel case. The same algorithms can readily be applied to the case of realistic slowly-fading timevarying channels. In the time-varying channel case, the transmission can be slotted and the cost function $\phi_{n}(\cdot)$ is a timevarying function given by (53) with the channels $H_{\mathrm{sd}}\left(e^{j \omega}\right)$, $H_{\mathrm{sr}}\left(e^{j \omega}\right)$ and $H_{\mathrm{rd}}\left(e^{j \omega}\right)$ at time slot $n$. With our APSM-based approach the "optimal" source and relay filters at time slot $n$ can simply be obtained as an updated version of that at time slot $n-1$. In this way, our APSM-based adaptive filter design approach reduces the computational complexity significantly compared with simple redesigning methods such as the interior point method in the time-varying channel case.

$$
\mathbf{u}_{n+1}= \begin{cases}P_{\xi_{\mathbf{h}}\left(P_{B_{\mathbf{t}}\left(P_{s}\right)}\left(\mathbf{t}_{n}\right)\right)}\left(\mathbf{h}_{n}\right) \circ P_{B_{\mathbf{t}}\left(P_{s}\right)}\left(\mathbf{t}_{n}\right) \circ\left(\mathbf{u}_{n}-\kappa_{n} \phi^{\prime}\left(\mathbf{u}_{n}\right)\right), & \text { if } \phi^{\prime}\left(\mathbf{u}_{n}\right) \neq 0 \\ \mathbf{u}_{n}, & \text { otherwise }\end{cases}
$$




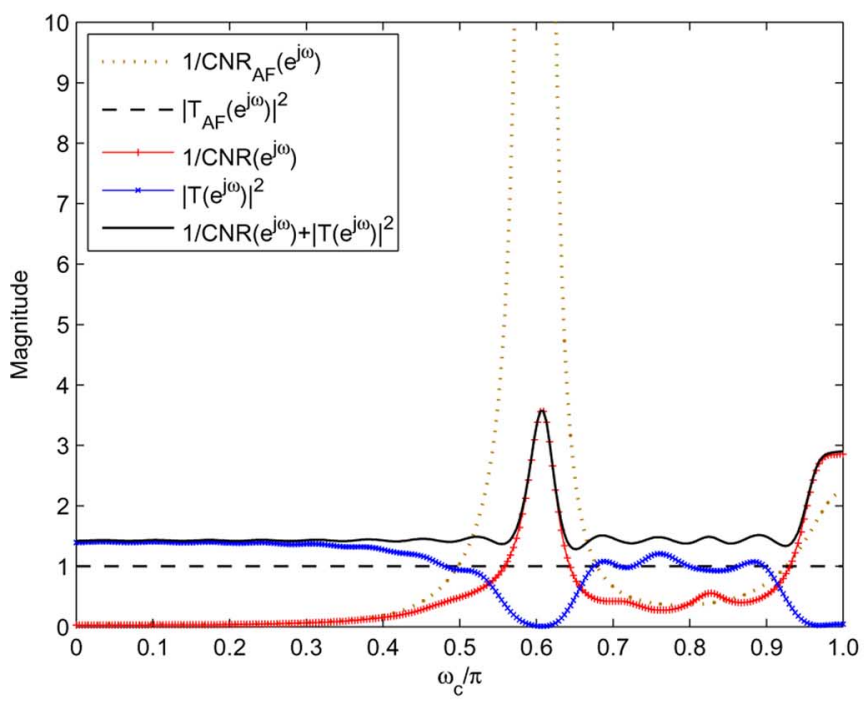

(a)
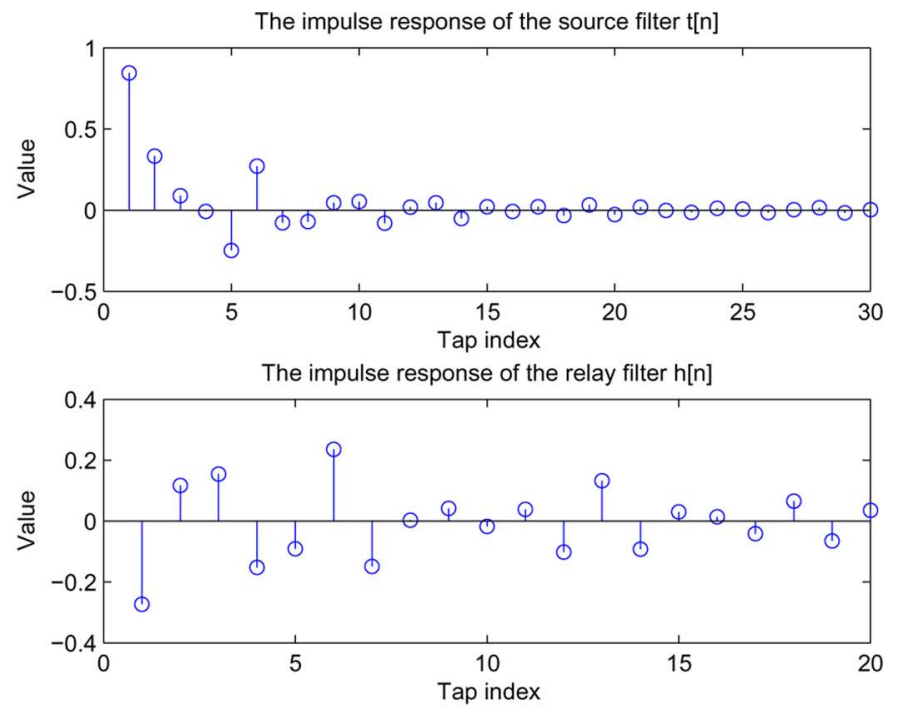

(b)

Fig. 8. (a) The frequency responses related to the proposed method. (b) The impulse responses related to the proposed method.

\section{NUMERICAL RESUlTS}

To evaluate the performance of the proposed joint filter design method presented in Section $\mathrm{V}$, we ran extensive simulations under various channel conditions. The channel order $L$ of all the propagation channels $H_{\mathrm{sr}}(z), H_{\mathrm{rd}}(z)$ and $H_{\mathrm{sd}}(z)$ was selected as $L=5$, and each channel tap coefficient was generated independently and identically distributedly (i.i.d.) according to a Gaussian distribution with a different variance for a different channel, i.e.,

$$
\begin{aligned}
& h_{\mathrm{sd}}[l] \stackrel{\text { i.i.d. }}{\sim} \mathcal{N}\left(0, \sigma_{\mathrm{sd}}^{2}\right), h_{\mathrm{sr}}[l] \stackrel{\text { i.i.d. }}{\sim} \mathcal{N}\left(0, \sigma_{\mathrm{sr}}^{2}\right) \text { and } \\
& h_{\mathrm{rd}}[l] \stackrel{\text { i.i.d. }}{\sim} \mathcal{N}\left(0, \sigma_{\mathrm{rd}}^{2}\right)
\end{aligned}
$$

for $l=0,1, \ldots, L-1$. Throughout the simulations, we fixed $\sigma^{2}=1$ and used $L_{s}=30$ and $L_{r}=20$ for the orders of the source and relay filters, respectively, to allow enough freedom for the two filters in the case of $L=5$. We used Algorithm 2 with the initial point ${ }^{5}$ (70) for the simulations, and the step size for the algorithm was adaptively changed according to $\kappa_{n}=\frac{1}{\sqrt{n}} \frac{\phi_{n}\left(\mathbf{u}_{n}\right)}{\left\|\phi_{n}^{\prime}\left(\mathbf{u}_{n}\right)\right\|^{2}}$ as the number of iterations increases. The stopping condition for the algorithm was either that the square of the normalized difference of two successive updates is less than $10^{-5}$ (i.e., $\frac{\left\|\mathbf{u}_{n+1}-\mathbf{u}_{n}\right\|^{2}}{\left\|\mathbf{u}_{n}\right\|^{2}} \leq 10^{-5}$ ) or that the number of iterations exceeds 1000 . For the numerical integration to compute the quantities $(53,54,58)$, we used a 512-point Gaussian quadrature method over $[-\pi, \pi]$. Since it is not clear how to optimally apply the known coding strategies such as DF and CF to the case of ISI channels, we do not consider these schemes in this section, and thus we use the AF scheme as the performance reference.

First, Fig. 8(a) shows the frequency responses related to the proposed method and Fig. 8(b) shows the corresponding impulse responses of source and relay filters obtained by Algorithm 2. The channel coefficients for this figure were gener-

\footnotetext{
${ }^{5}$ We observed that Algorithm 2 with the initialization (64) did not make a noticeable difference.
}

ated randomly according to $\sigma_{\mathrm{sr}}^{2}=\sigma_{\mathrm{rd}}^{2}=\sigma_{\mathrm{sd}}^{2}=1$ and given by $H_{\mathrm{sr}}=1.8833+0.3254 z^{-1}-0.0952 z^{-2}+0.0312 z^{3}-$ $0.6138 z^{-4}, H_{\mathrm{rd}}=-0.0728+1.3148 z^{-1}+0.9783 z^{-2}+$ $1.7221 z^{3}-0.4123 z^{-4}$ and $H_{\mathrm{sd}}=-0.8864-1.8402 z^{-1}-$ $1.6282 z^{-2}-1.1738 z^{3}-0.4154 z^{-4} . P_{s}=P_{r}=1$. The flat ${ }^{6}$ dashed line represents the input spectrum of the conventional AF scheme, and the dotted line is the inverse of the CNR density, i.e., the effect noise level, in the case that the AF scheme is used at the relay. It is seen that there are two peaks in the noise level for the AF scheme around the normalized frequency values of 0.6 and 1 . Note that the peak around 0.6 is very high and its width is also large, whereas the peak around 1 is mild. Now the red solid line marked with + denotes the effective noise level generated by the relay filter designed by the proposed method. We recognize that the designed relay filter is smart. One could imagine that a reasonable relay filter would suppress the frequency band having a bad overall response with the AF scheme and enhance the frequency band having a good overall response with the AF scheme so that the water-filling by the source filter might be enhanced. This is exactly the case with the mild noise peak around 1; the designed relay filter reinforces the mild noise peak. However, the designed relay filter suppresses the severe noise peak with a large width around 0.6 instead of reinforcing it. This is because the width of the severe peak is large and, thus, the loss of this frequency band will reduce the transmission rate. To this optimally shaped noise level, the water-filling-type source power allocation is performed by the designed source filter satisfying both the source and relay power constraints jointly with the designed relay filter; for the

${ }^{6}$ The reason why the flat input spectrum is used for the conventional AF scheme is that even for a given relay filter, e.g., the AF filter, the optimal power allocation to maximize the transmission rate (15) not only with the source power constraint (17) but also with the relay power constraint (18) in ISI channels is not a trivial problem; this is not a simple and known water-filling problem with a single total power constraint because of the term $\left|H_{\mathrm{sr}}\left(e^{j \omega}\right)\right|^{2}$ in (18) unless $H_{\mathrm{sr}}\left(e^{j \omega}\right)$ is constant over $[-\pi, \pi]$. As far as we know, this problem was not handled before, and the two-step projection method in this paper provides one way to accomplish this in general ISI channels. 


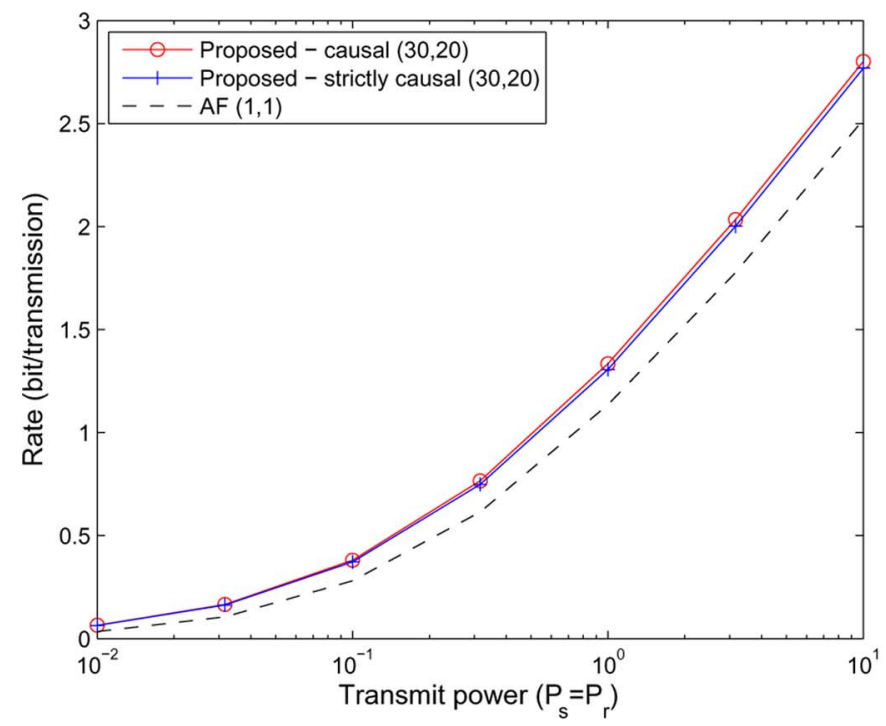

(a)

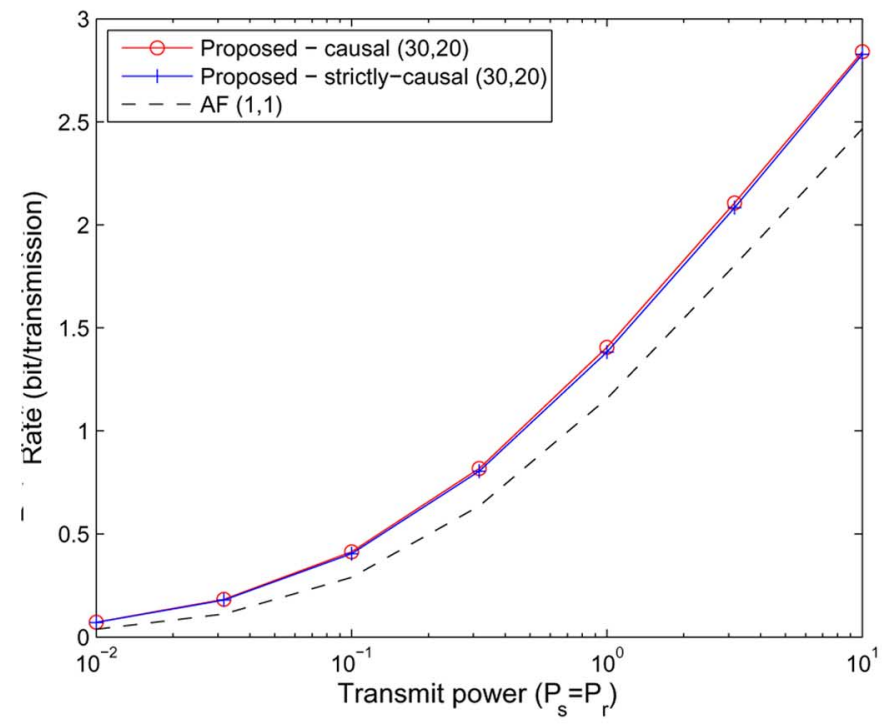

(c)

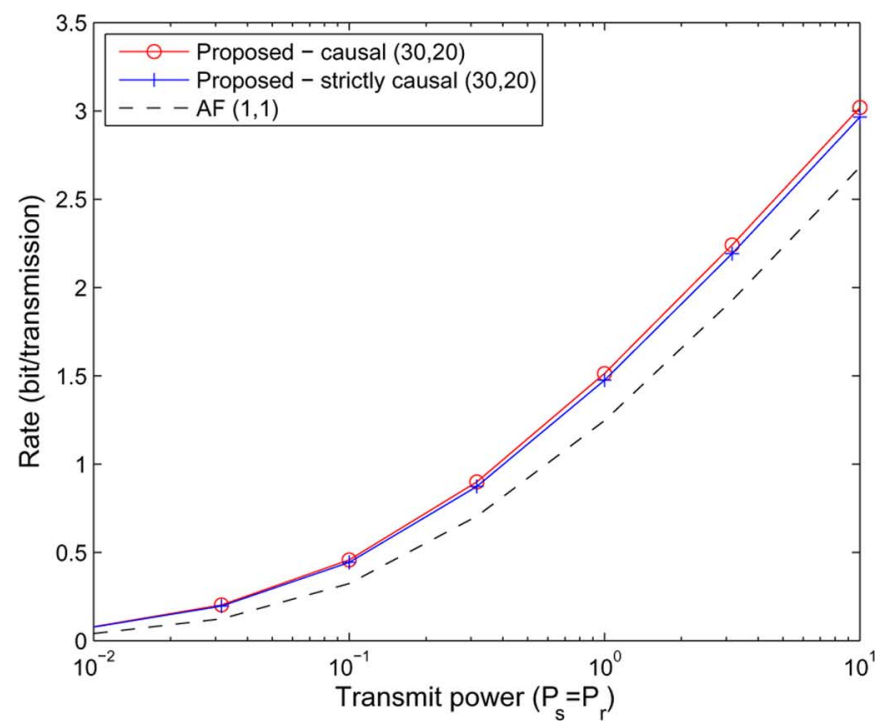

(b)

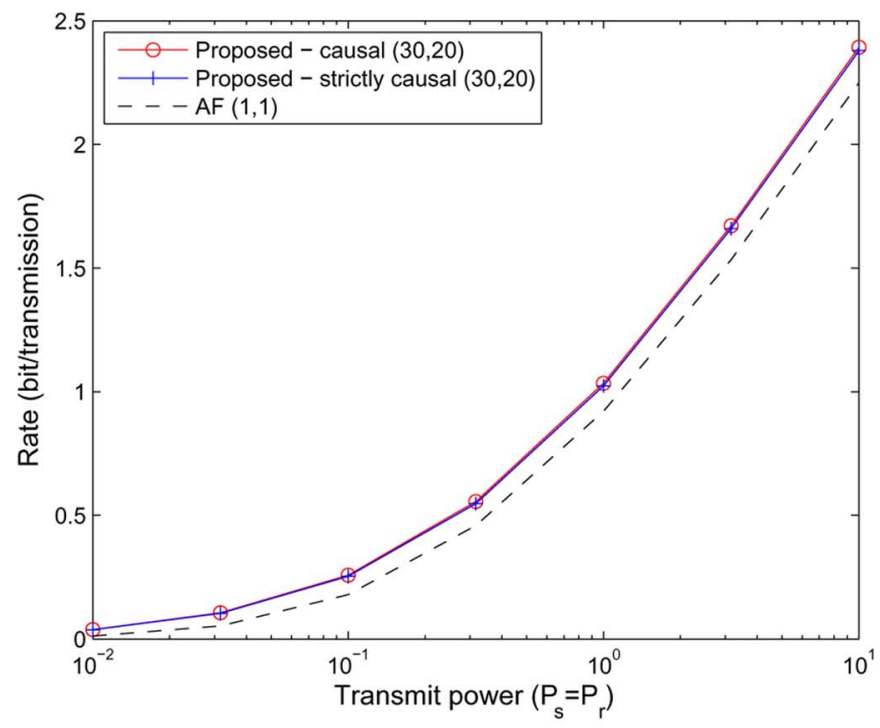

(d)

Fig. 9. Transmission rate averaged over channel realizations $\left(P_{s}=P_{r}\right)$ : (a) $\sigma_{\mathrm{sd}}^{2}=1, \sigma_{\mathrm{sr}}^{2}=1, \sigma_{\mathrm{rd}}^{2}=1$, (b) $\sigma_{\mathrm{sd}}^{2}=1, \sigma_{\mathrm{sr}}^{2}=4, \sigma_{\mathrm{rd}}^{2}=1,(\mathrm{c}) \sigma_{\mathrm{sd}}^{2}=1, \sigma_{\mathrm{sr}}^{2}=1$, $\sigma_{\mathrm{rd}}^{2}=4$, and (d) $\sigma_{\mathrm{sd}}^{2}=\frac{1}{10}, \sigma_{\mathrm{sr}}^{2}=1, \sigma_{\mathrm{rd}}^{2}=10$.

frequency bands around the two noise peaks the power is not allocated, as seen in the figure. (See the green solid line without any markers.) Similar behaviors are observed in other settings even though they are not shown in this paper.

Next, let us see the performance of the proposed method in various channel settings. Fig. 9 shows the transmission rate achieved by the proposed method averaged over 100 independent channel realizations for each of the different channel gain settings when $P_{s}=P_{r}$. We considered both causal and strictly causal filtering for the proposed method. For the strictly causal filtering, we eliminated the $t_{0}$ and $h_{0}$ terms in the formulation and kept the same filter orders $L_{s}$ and $L_{r}$ as those in the causal filtering case. We considered two sets of channel conditions; in one set the direct S-D link has a reasonable strength compared with the S-R and R-D links [Fig. 9(a), (b) and (c)], and in the other set the direct link is weak compared with the S-R and R-D links [Fig. 9(d)]. (We did not consider the case that the direct link is stronger than the S-R and R-D links since it is unnecessary to use the relay in this case.) It is seen in Fig. 9 that the gain obtained by the proposed joint source and relay filtering over the AF scheme is considerable. In particular, Fig. 9(c) shows the performance when $P_{s}=P_{r}, \sigma^{2}=1, \sigma_{\mathrm{sd}}^{2}=1, \sigma_{\mathrm{sr}}^{2}=1$ and $\sigma_{\mathrm{rd}}^{2}=4$, which is equivalent to the flat-fading case of $a=1$ and $b=2$ in Fig. 2(a) and (b) and 4. Compared with the flat-fading case shown in Fig. 4(c), the gain by the proposed joint design over the AF scheme in ISI channels is significant. It is also seen that the loss caused by the strict causality of filtering is not significant in the proposed scheme. Fig. 9(d) shows the case that the direct link is weak compared with the S-R and R-D links. The gain in this case is smallest among the four cases. This is explained as follows. As seen in $(15,16)$, the gain of the joint filtering depends on various factors. The worst situation for the performance of the joint filtering is the case that $H_{\mathrm{sd}}\left(e^{j \omega}\right) \approx 0$ and $H_{\mathrm{rd}}\left(e^{j \omega}\right) \gg 0$ such that $\left|H_{\mathrm{rd}}\left(e^{j \omega}\right) H\left(e^{j \omega}\right)\right| \gg 1$. In this 


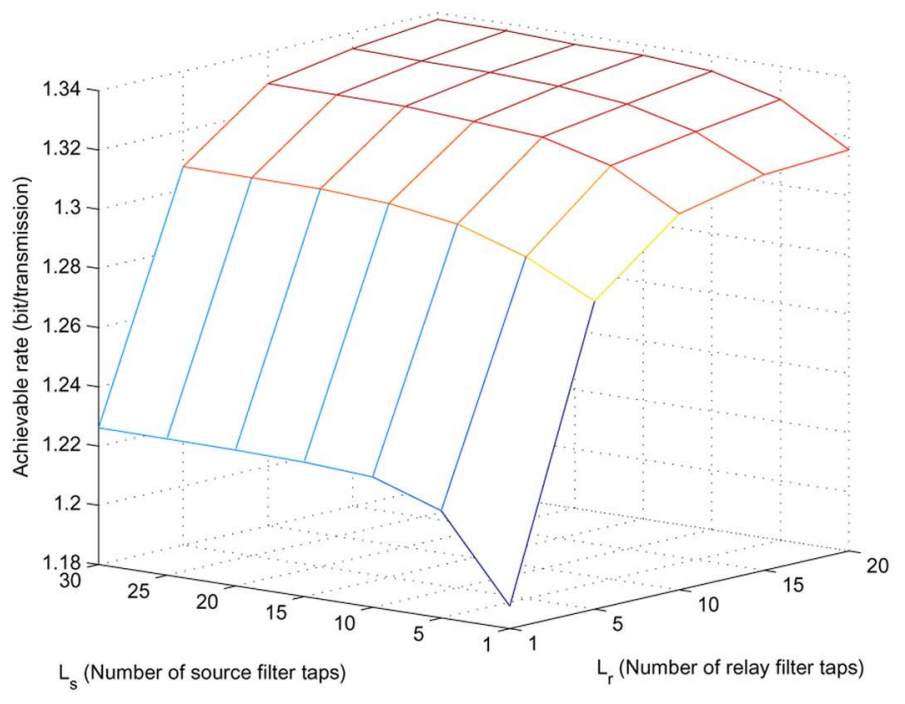

(a)

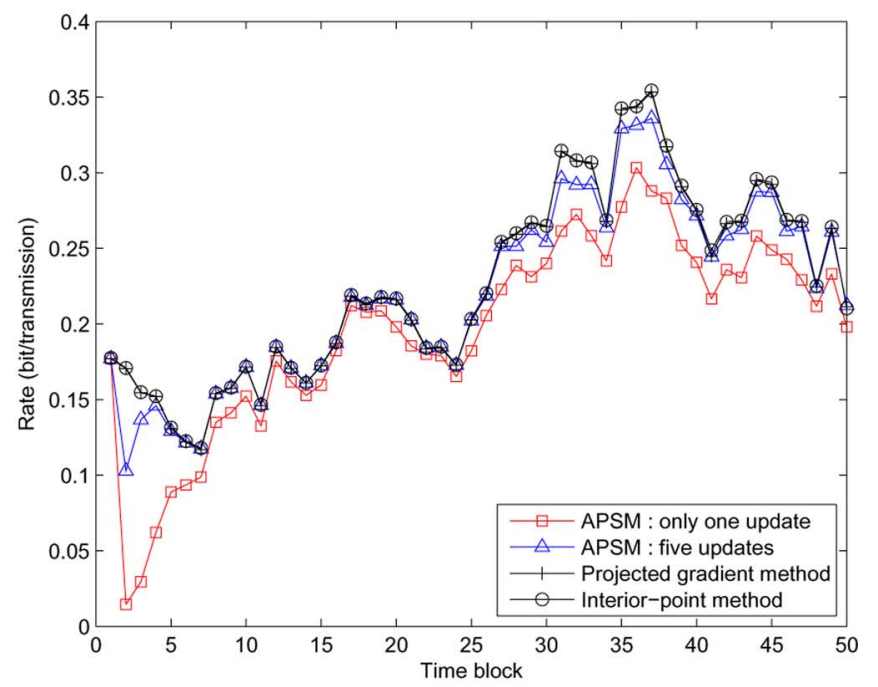

(b)

Fig. 10. (a) achievable rate with respect to the length of filters and (b) achievable rates of several schemes for the blockwise first-order Gaussian AR fading channel model.

case, the CNR density in (16) is approximated by $\operatorname{CNR}\left(e^{j \omega}\right) \approx$ $\frac{\left|H_{\mathrm{sr}}\left(e^{j \omega}\right) H\left(e^{j \omega}\right) H_{\mathrm{rd}}\left(e^{j \omega}\right)\right|^{2}}{\left(\sigma^{2}\left|H_{\mathrm{rd}}\left(e^{j \omega}\right) H\left(e^{j \omega}\right)\right|^{2}\right)}=\frac{\left|H_{\mathrm{sr}}\left(e^{j \omega}\right)\right|^{2}}{\sigma^{2}}$; and thus the impact of the relay filter disappears and only the optimal power allocation by the source filter under the two power constraints is effective. Even in this case, a non-negligible gain is observed. Although the results in the cases of $P_{s}=2 P_{r}$ and $2 P_{s}=P_{r}$ are not shown in this paper, we observed similar results to the case of $P_{s}=P_{r}$. We also investigated the performance w.r.t. the filter length. Here, we swept the lengths $\left(L_{s}, L_{r}\right)$ of the source and relay filters from $(1,1)$ to $(30,20)$, and the result is shown in Fig. 10(a). It is seen that the impact of the relay filter length is more important to the rate performance than that of the source filter length. The reasonable value for filter length depends on the channel tap number, but for the considered 5 tap case $\left(L_{s}, L_{r}\right)=(10,10)$ already attains a significant portion of the maximum performance.

Finally, the performance of our APSM-based rate-maximizing filter design algorithm is investigated in a time-varying scenario. The destination node is assumed to move at the speed of $3 \mathrm{~km} / \mathrm{h}$ while the source and relay nodes are stationary. This is a reasonable assumption when the source node is a basestation and a fixed relay is deployed to enlarge the cell coverage. Each of the S-D, S-R, R-D ISI channels is a 5-tap FIR filter, and the S-D and R-D channels are time-varying. (In this case, the feasible set for the power constraints does not time-varying.) The widely-used first-order Gaussian autoregressive (AR) channel process is used for each tap of the time-varying S-D and R-D channel filters with $\sigma_{\text {sd }}^{2}=\frac{1}{10}$ and $\sigma_{\text {rd }}^{2}=10$. For the static S-R channel each channel tap coefficient is realized once according to $h_{\mathrm{sr}} \sim N(0,1)$ and is kept throughout the time slots. The AR coefficient is determined by $J_{0}\left(2 \pi f_{D} n T_{s}\right)$, where $J_{0}(\cdot)$ the Bessel function of the first kind, $f_{D}$ is the Doppler frequency, $T_{s}=66.7 \mu \mathrm{s}$ is the 3GPP symbol duration and $n=100$ is the number of symbols in one block or slot. The power budget is $P_{s}=P_{r}=1$. Fig. 10(b) shows the achievable rates of four filter design methods in this time-varying scenario. For the first two schemes, the projected gradient method and the interior point method given by the MATLAB function fmincon are applied to each block afresh until they converge. As shown in Fig. 10(b), these two methods yield almost the same performance. The next two methods are two adaptive approaches based on APSM-based Algorithm 2 in Section V-B. First we apply only one update per each time block with $\kappa_{n}=\frac{\phi_{n}\left(\mathbf{u}_{n}\right)}{\left\|\phi_{n}^{\prime}\left(\mathbf{u}_{n}\right)\right\|^{2}}$, and next we apply five updates per time block. It is seen that the proposed adaptive method tracks the rate very well. It shows the steady-state behavior after only a few time blocks. Even with only one update it can still track the "best" rate with an admissible performance loss.

\section{CONCLUSION}

We have considered the linear Gaussian relay problem. By adopting the LTI relay filtering and realizable input spectra, we have converted the rate maximization problem to the problem of joint source and relay filter design. We have investigated the performance of this joint LTI filtering in flat-fading relay channels, and have shown the optimality of the AF scheme within the class of one-tap filters. In general ISI relay channels, we have developed a practical method for the joint filter design to maximize the transmission rate based on the adaptive projected subgradient method under the linear FIR filtering framework, and have shown numerically that the gain of the proposed design is considerable compared with the AF scheme in ISI relay channels.

\section{ACKNOWLEDGMENT}

The authors of this paper would like to thank G. Lee, M. Yukawa, and I. Yamada for their introduction to the adaptive projected subgradient method, and anonymous reviewers for their valuable comments.

\section{REFERENCES}

[1] T. M. Cover and A. El Gamal, "Capacity theorems for the relay channel," IEEE Trans. Inf. Theory, vol. 25, pp. 572-584, Sep. 1979. 
[2] A. El Gamal and M. Aref, "The capacity of the semideterministic relay channel," IEEE Trans. Inf. Theory, vol. 28, pp. 536-536, May 1986.

[3] S. Zahedi, M. Mohseni, and A. El Gamal, "On the capacity of AWGN relay channels with linear relaying functions," in Proc. ISIT, Chicago, IL, Jun. 2004.

[4] A. El Gamal, M. Mohseni, and S. Zahedi, "Bounds on capacity and minimum energy-per-bit for AWGN relay channels," IEEE Trans. Inf. Theory, vol. 52, pp. 1545-1561, Apr. 2006.

[5] G. Kramer, M. Gastpar, and P. Gupta, "Cooperative strategies and capacity theorems for relay networks," IEEE Trans. Inf. Theory, vol. 51, pp. 3037-3063, Sep. 2005.

[6] A. El Gamal, N. Hassanpour, and J. Mammen, "Relay networks with delays," IEEE Trans. Inf. Theory, vol. 53, pp. 3413-3431, Oct. 2007.

[7] A. del Coso and C. Ibars, "Achievable rates for the AWGN channel with multiple parallel relays," IEEE Trans. Wireless Commun., vol. 8, pp. 2524-2534, May 2009.

[8] M. N. Khormuji and M. Skoglund, "On instantaneous relaying," IEEE Trans. Inf. Theory, vol. 56, pp. 3378-3394, Jul. 2010.

[9] H. Chen, A. B. Gershman, and S. Shahbazpanahi, "Filter-and-forward distributed beamforming in relay networks with frequency selective fading," IEEE Trans. Signal Process., vol. 58, pp. 1251-1262, Mar. 2010.

[10] Y.-W. Liang, A. Ikhlef, W. Gerstacker, and R. Schober, "Cooperative filter-and-forward beamforming for frequency-selective channels with equalization," IEEE Trans. Wireless Commun., vol. 10, pp. 228-239, Jan. 2011.

[11] A. S. Behbahani, R. Merched, and A. M. Eltawil, "Optimizations of a MIMO relay network," IEEE Trans. Signal Process., vol. 56, no. 10, pp. 5062-5073, Oct. 2008.

[12] R. Mo and Y. H. Chew, "MMSE-based joint source and relay precoding design for amplify-and-forward MIMO relay networks," IEEE Trans. Wireless Commun., vol. 8, no. 9, pp. 4668-4676, Sep. 2009.

[13] Z. Fang, Y. Hua, and J. C. Koshy, "Joint source and relay optimization for a non-regenerative MIMO relay," in Proc. IEEE SAM, 2006.

[14] S. Simoens, O. Munoz-Medina, J. Vidal, and A. del Coso, "On the Gaussian MIMO relay channel with full channel state information," IEEE Trans. Signal Process., vol. 57, no. 9, pp. 3588-3599, Sep. 2009.

[15] U. Grenander and G. Szegö, Toeplitz Forms and Their Applications. Berkeley, CA: Univ. of California Press, 1958.

[16] P. J. Brockwell and R. A. Davis, Time Series: Theory and Methods, 2nd ed. New York: Springer, 1991

[17] R. G. Gallager, Information Theory and Reliable Communication. New York: Wiley, 1968.

[18] A. A. Goldstein, "Convex programming in Hilbert space," Bull. Amer. Math. Soc. 70, pp. 709-710, 1964.

[19] B. T. Polyak, "Minimization of unsmooth functionals," USSR Comput. Math. Phys., vol. 9, pp. 14-29, 1969.

[20] I. Yamada and N. Ogura, "Adaptive projected subgradient method for asymptotic minimization of sequence of nonnegative convex functions," Numer. Funct. Anal. Optim., vol. 25, no. 7-8, pp. 593-617, 2004.

[21] K. Slavakis, I. Yamada, and N. Ogura, "The adaptive projected subgradient method over the fixed point set of strongly attracting nonexpansive mappings," Numer. Funct. Anal. Optim., vol. 27, no. 7, pp. 905-930, 2006.

[22] T. Kailath, A. H. Sayed, and B. Hassibi, Linear Estimation. Upper Saddle River, NJ: Prentice-Hall, 2000.

[23] T. Cover and J. Thomas, Elements of Information Theory, 2nd ed. New York: Wiley, 2006.

[24] S. Boyd and L. Vandenberghe, Convex Optimization. New York: Cambridge Univ. Press, 2004.

[25] S. Theodoridis, K. Slavakis, and I. Yamada, "Adaptive learning in a world of projections," IEEE Signal Process. Mag., vol. 28, pp. 97-123, Jan. 2011.
[26] Y. Censor and S. A. Zenios, Parallel Optimization: Theory, Algorithms and Applications. New York: Oxford Univ. Press, 1997.

[27] , H. H. Bauschke, R. Burachik, P. L. Combettes, V. Elser, D. R. Luke, and H. Wolkowicz, Eds., Fixed-Point Algorithms for Inverse Problems in Science and Engineering. New York: Springer, 2011.

[28] Y. N. Kiseliov, "Algorithms of projection of a point onto an ellipsoid," Lithuanian Math. J., vol. 34, no. 2, pp. 141-159, 1994

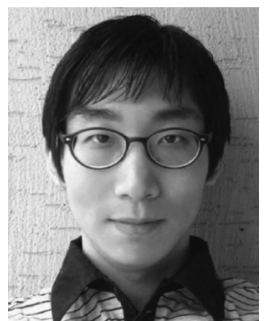

Cheulsoon Kim (S'09) received the B.S. degree from Pohang University of Science and Technology (POSTECH), Pohang, Korea, in 2007 and the M.S. degree from Korea Advanced Institute of Science and Technology (KAIST), Daejeon, Korea, in 2009 , both in electrical engineering. He is currently working toward the Ph.D. degree in the Department of Electrical Engineering, KAIST.

His research interests include statistical signal processing and communication theory.

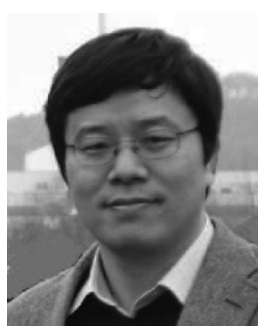

Youngchul Sung (S'92-M'93-SM'09) received the B.S. and M.S. degrees in electronics engineering from Seoul National University, Seoul, Korea, in 1993 and 1995, respectively, and the Ph.D. degree in electrical and computer engineering from Cornell University, Ithaca, NY, in 2005.

From 2005 until 2007, he was a Senior Engineer in the Corporate R\&D Center of Qualcomm, Inc., San Diego, CA, and also a Senior Research Engineer in LG Electronics, Ltd., Seoul, Korea, from 1995 to 2000. He is currently an Associate Professor in the Department of Electrical Engineering in the Korea Advanced Institute of Science and Technology (KAIST), Daejeon, Korea. His research interests include signal processing for communications, statistical signal processing, and asymptotic statistics with applications to wireless communications and related areas.

Dr. Sung is an Associate Member of the IEEE Signal Processing Society SPCOM Technical Committee, a member of the Signal and Information Processing Theory and Methods (SIPTM) Technical Committee of the Asia-Pacific Signal and Information Processing Association (APSIPA), a Technical Program Committee member of Globecom 2011/2010/2009, ICC 2011, MILCOM 2010 , DCOSS 2010, WiOpt 2009 and its sponsorship chair, APSIPA 2010/2009, IEEE SAM 2008.

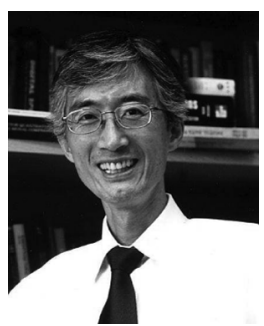

Yong H. Lee (S'81-M'84-SM'98) was born in Seoul, Korea, on July 12, 1955. He received the B.S. and M.S. degrees in electrical engineering from Seoul National University, Seoul, Korea, in 1978 and 1980, respectively, and the Ph.D. degree in electrical engineering from the University of Pennsylvania, Philadelphia, in 1984

From 1984 to 1988, he was an Assistant Professor with the Department of Electrical and Computer Engineering, State University of New York, Buffalo. Since 1989, he has been with the Department of Electrical Engineering, Korea Advanced Institute of Science and Technology (KAIST), Daejeon, Korea, where he is currently a Professor and the Provost of KAIST. His research activities are in the area of communication signal processing, which includes interference management, resource allocation, synchronization and estimation for OFDM/MIMO systems with relays. 